\title{
Bounded variation singular stochastic control and associated Dynkin game
}

\author{
Frederik Boetius*
}

March 21, 2000

\begin{abstract}
We consider an optimal control problem for a one-dimensional Itô diffusion and a stochastic game of optimal stopping associated with it. Their value functions satisfy $\frac{\partial}{\partial x} V=u$ and an optimal control defines a saddle point for the game. This extends earlier results to the case of bounded variation control and general nonadditive cost functionals in the form of a controlled FBSDE. Our approach uses probabilistic methods such as comparison theorems, and a pathwise construction of policies.
\end{abstract}

AMS 2000 subject classification Primary 60G40, 60H10, 93E20; Secondary $91 \mathrm{~A} 15$

Key words and phrases Backward stochastic differential equation; Singular stochastic control; Optimal stopping; Dynkin games; Adjoint equation; Comparison theorem

\section{Contents}

1 Introduction 3

1.1 Related literature . . . . . . . . . . . . . . . . . 3

1.2 Organization of this paper $\ldots \ldots \ldots \ldots \ldots \ldots$

2 Preliminaries 6

2.1 Problem formulation . . . . . . . . . . . . . . 6

2.2 Definition of control problem and Dynkin game . . . . . . . 8

2.3 Existence and uniqueness of solutions . . . . . . . . . 9

2.4 A priori estimate and comparison theorem for forward equation . 10

2.5 A priori estimates and Comparison Theorem for BSDE . . . . . . 13

2.6 Properties of the value function . . . . . . . . . . . . . 16

2.7 Properties of controlled forward processes . . . . . . . . . 19

3 Deriving the stopping problem 22

3.1 Upper right Dini derivative . . . . . . . . . . . . . . . 23

3.2 Lower left Dini derivative . . . . . . . . . . . . . . . . 34

3.3 Proof and discussion . . . . . . . . . . . . . . . 39

${ }^{*}$ Centre of Finance and Econometrics, Universität Konstanz, 78434 Konstanz, Germany 
4 Characteristic RBSDE

References 


\section{Introduction}

The theory of backward stochastic differential equations (BSDE) is by now a primary tool in mathematical finance. Its applications range from the term structure of interest rates to pricing and hedging of contingent claims, covering problems such as mean variance hedging, imperfect markets, large investor, american contingent claims and stochastic Black-Scholes formulae. The theory originates from stochastic control theory, where BSDE arise as adjoint equations in the Pontryagin maximum principle. In turn, stochastic control problems of a "singular" type have applications in mathematical finance in the context of irreversible investment and real options or, generally speaking, imperfect markets with decision rigidities such as hedging or portfolio optimization under transaction costs.

Our reformulation of a singular control problem as optimization problem for decoupled FBSDE is motivated by the observation - yet present in derivations of the classic (deterministic) Black-Scholes formula - that the value of a dynamic optimization problem is actually a stochastic process, and this is determined by the future development of the stochastic system. The FBSDE formulation is also necessary to allow for the use of nonadditive or stochastic differential utilities and $g$-expectation in the control problem. In this formulation we establish a relation between singular stochastic control and optimal stopping, namely that the partial derivative of the value of the control problem $\frac{\partial}{\partial x} V$ equals the value of a stochastic game of optimal stopping $u$, and a solution to this game can be derived from an optimal control in the control problem.

This relation is well known in special cases and gives valuable insights into both problems, among them the old question of the relation between the principle of smooth fit in control and the smooth pasting condition in optimal stopping. In our present paper, we extend it to the case of a general Brownian diffusion in one dimension, with bounded variation instead of monotone controls. This is the first step in establishing the equivalence of both problems in the sense of Baldursson and Karatzas [6], Boetius and Kohlmann [12]. Together with recent results on the relation between reflected backward stochastic differential equations (RBSDE) and optimal stopping we propose that the adjoint equation for a singular stochastic control problem has the form of a RBSDE.

We also note that the controlled BSDE in our formulation exhibits a formal similarity with solutions of RBSDE and $g$-semisolutions in the sense of PENG [48], [49]. To arrive at our results we make use of methods from stochastic analysis, especially comparison theorems for the solutions of controlled forward and backward stochastic differential equations.

\subsection{Related literature}

The theory of adapted solutions to BSDE was brought to life with the fundamental paper of PARDoux and Peng [47] and independently by DufFie and EPSTEIN [19] and has received much attention since then. Yong and ZHOU [58] give a recent account of the theory and its development. Reflected BSDE were introduced in El Karoui, Kapoudjian, Pardoux, Peng and Quenez [21]. Solvability of coupled FBSDE was first studied by ANTONELLi [3] over short time durations and solved in for arbitrary durations through the four-step- 
scheme by MA, Protter and Yong [41]. The subject, including applications in finance, is presented in great depth in MA and YoNG [42]. An overview over applications in finance can be found also in EL KarouI and Quenez [24]. The whole field remains very active, with the number of publications still increasing.

The study of singular stochastic control problems dates back to an investigation of spaceship control by BATHER and CHERNOFF [7], who also noted the connection with a problem of optimal stopping and the relation $\frac{\partial}{\partial x} V=u$. Subsequently this link has been studied in a number of different settings. Besides the monotone follower problem for Brownian motion these include reflection and absorption, bounded variation follower and additional finite fuel condition.

There are two main approaches to the link between problems of optimal stopping and singular stochastic control. One of them makes use of analytical properties of the value functions, usually including partial differential equations techniques and characterizations of value functions through variational inequalities. Various aspects are described in e.g. Alvarez [2], Arntzen [4], Chow, Menaldi and Robin [13], Davis and Norman [17], Menaldi and Taksar [44], Karatzas and Shreve [37], Karatzas [32], [33]. A younger approach was initiated by Karatzas and Shreve [35], [36]; their pathwise comparison forms the basis of our argument in section 3. Probabilistic arguments were also used by several other authors, among them BALDURSSON [5], BoETIUS and Kohlmann [12], El Karoui and Karatzas [22], [23], Karatzas [34]. Our derivation of the relation between optimal control and optimal stopping is along the lines of [35].

Singular stochastic control problems where further treated in BENEŠ, SHEPP and Witsenhausen [8], Shreve, Lehotzky and Gaver [54], Shreve [53], Soner and Shreve [55], including aspects of higher dimensions and properties of the value function. Applications to irreversible investment, industry equilibrium and portfolio optimization under transaction costs can be found in Baldursson and Karatzas [6], Kobila [39] and Davis and Norman [17]. The latter provide a solution to a two-dimensional control problem including singular and absolutely continuous control.

Treatment of hedging and portfolio optimization under transaction costs using Martingale and other methods was carried out in Soner, Shreve and Cvitanić [56], Cvitanić, Pham and Touzi [16] and Cvitanić and Karatzas [15]. The economics of irreversible investment are dicussed e.g. in MCDonald and Siegel [43] and PINDYCK [50]; an extensive account of investment under uncertainty is given in DIXIT and PINDYCK [18].

There is a vast literature on optimal stopping problems. Standard references include Friedman [26] and Shiryayev [52]. Games of stopping, as correspondent to the bounded variation control, have been studied in DyNKIN and Yushkevich [20], Neveu [46], Bensoussan and Friedman [9], Bismut [10], Stettner [57], Morimoto [45], Alario-Nazaret, Lepeltier and MarCHAL [1] and others.

Stochastic games in the context of backward equations are studied in HAMADene, Lepeltier and Peng [30], Hamadene and Lepeltier [27], [28]. The observation that a solution to a reflected BSDE also solves an optimal stopping problem is due to El Karoui, Kapoudjian, Pardoux, Peng and Quenez [21] and was extended to double barrier reflection and stochastic games by CVITANIĆ and KARATZAS [14]. 


\subsection{Organization of this paper}

In section 2 we define the control problem and associated stochastic game of optimal stopping, introduce the comparison theorems and a priori estimates central to our approach and present some preliminary results on the controlled process plus convexity of solutions and the value function. In section 3 we formulate and prove the main result of this paper. We also discuss some extensions, related problems and the route to equivalence of singular control and optimal stopping. In section 4 we motivate the proposition that singular control problems have RBSDE as adjoint equations. 


\section{Preliminaries}

In this section we introduce a controlled forward-backward SDE, the control problem and its associated stochastic game of optimal stopping. We also recall some properties of controlled processes and prove convexity of the value function of the control problem.

\subsection{Problem formulation}

Let $(\Omega, \mathcal{F}, \boldsymbol{P})$ be a probability space, $\bar{\imath}:=[0, T]$ a time interval, and $W_{t}$ a standard $d$-dimensional Brownian motion adapted to a filtration $\left\{\mathcal{F}_{t}\right\}_{t \in \bar{\imath}}$ satisfying the usual conditions.

We use the following notation as defined in [42] for the different spaces of measurable random variables:

- for a $\sigma$-algebra $\mathcal{G} \subset \mathcal{F}_{T} L_{\mathcal{G}}^{p}\left(\Omega ; \mathbb{R}^{n}\right)$ denotes the set of $\mathcal{G}$-measurable $\mathbb{R}^{n}$ valued random variables $X$ such that $E_{\boldsymbol{P}}\left[|X|^{p} d t\right]<\infty$.

- $L_{\mathcal{F}}^{p}\left(\Omega ; L^{p}\left(0, T ; \mathbb{R}^{n}\right)\right)$ the set of $\mathcal{F}_{t}$-progressively measurable $\mathbb{R}^{n}$-valued processes $X_{t}$ such that $E_{\boldsymbol{P}}\left[\int_{0}^{T}\left|X_{t}\right|^{p}\right]<\infty$; we write $L_{\mathcal{F}}^{p}\left(0, T ; \mathbb{R}^{n}\right)$ if there is no danger of confusion.

- $L_{\mathcal{F}}^{p}\left(\Omega ; C\left(\bar{\imath} ; \mathbb{R}^{n}\right)\right)$ the set of $\mathcal{F}_{t}$-progressively measurable continuous $\mathbb{R}^{n}$ valued processes $X_{t}$ such that $E_{\boldsymbol{P}}\left[\sup _{\bar{\imath}}\left|X_{t}\right|^{p}\right]<\infty$

- $L_{\mathcal{F}}^{p}\left(0, T ; W^{1, \infty}(M, N)\right)$ for Euclidean spaces $M, N$ the set of functions $f$ : $\bar{\imath} \times M \times \Omega \rightarrow N$ such that (a) for fixed $m \in M,(t, \omega) \mapsto f(t, m, \omega)$ is $\mathcal{F}_{t^{-}}$ progressively measurable, (b) $f(t, 0, \omega) \in L_{\mathcal{F}}^{p}(0, T ; N)$ and (c) there exists a constant $L \in \mathbb{R}_{>0}$ such that

$$
\left|f(t, m, \omega)-f\left(t, m^{\prime}, \omega\right)\right| \leq L\left|m-m^{\prime}\right|, \quad \forall m, m^{\prime} \in M \text {, a.e. } t \in \bar{\imath}, \boldsymbol{P} \text {-a.s. }
$$

- $L_{\mathcal{F}_{T}}^{p}\left(\Omega ; W^{1, \infty}(M, N)\right)$ for Euclidean spaces $M, N$ the set of functions $f$ : $M \times \Omega \rightarrow N$ such that for any $m \in M, \omega \mapsto f(m, \omega)$ is $\mathcal{F}_{T}$ measurable, $m \mapsto f(m, \omega)$ is uniformly Lipschitz and $f(0, \omega) \in L_{\mathcal{F}}^{p}(\Omega ; N)$.

$\|\cdot\|_{p}$ denotes the usual $p$-norm in the spaces defined above.

For a $\mathbb{R}^{n}$-valued process $B=\left(B^{j}\right)_{1 \leq j \leq n}^{T}$ of bounded variation its absolute is denoted by $|B|:=\sum_{j=1}^{n}\left|B^{j}\right|$; i.e. if $\mu_{j}=\mu_{j}^{+}-\mu_{j}^{-}$is the ( $\omega$-dependent) signed measure (with Hahn-decomposition $\left(\mu_{j}^{+}, \mu_{j}^{-}\right)$) defined by

$$
\mu_{j}(A):=\int \chi_{A} d B^{j} \quad \text { then } \quad \int \chi_{A} d|B|=\sum_{j=1}^{n}\left(\mu_{j}^{+}(A)+\mu_{j}^{-}(A)\right) .
$$

For a $\mathbb{R}^{n}$-valued progressively measurable process $a=\left(a^{j}\right)_{1 \leq j \leq n}$ and a $\mathbb{R}^{n}$ valued bounded variation process $C=\left(C^{j}\right)_{1 \leq j \leq n}$ we use - depending on our focus on a norm for $a$ or $C$ - the notation

$$
\|a\|_{[C, \bar{\imath}]}^{2}:=E\left[\sum_{j=1}^{n}\left(\int_{\bar{\imath}}\left|a_{s}^{j}\right| d\left|C^{j}\right|_{s}\right)^{2}\right]=:|C|_{[a, \bar{\imath}]}^{2}
$$


Remark 2.1. With the above definition and using the Hölder inequality $\|f g\|_{1} \leq$ $\|f\|_{2}\|g\|_{2}$ with $f=1$ and $g=a$ we have the estimates

$$
\begin{aligned}
E\left[\left(\int_{\bar{\imath}} a_{s}^{\top} d C_{s}\right)^{2}\right] & \leq 2\|a\|_{[C, \bar{\imath}]}^{2}=2|C|_{[a, \bar{l}]}^{2} \\
& \leq 2 E\left[\sum_{j=1}^{n}\left(\left|C^{j}\right|_{T} \int_{\bar{\imath}}\left|a^{j}(s)\right|^{2} d\left|C^{j}\right|_{s}\right)\right] .
\end{aligned}
$$

For a function $f: \mathbb{R} \rightarrow \mathbb{R}$ we define the Dini derivatives

$$
\Delta^{ \pm} f(x):=\limsup _{\delta \searrow 0} \frac{f(x \pm \delta)-f(x)}{ \pm \delta}, \quad \Delta_{ \pm} f(x):=\liminf _{\delta \searrow 0} \frac{f(x \pm \delta)-f(x)}{ \pm \delta}
$$

and the one-sided differentials $D^{+} f:=\Delta^{+} f=\Delta_{+} f$ and $D^{-} f:=\Delta^{-} f=\Delta_{-} f$, if they exist. Note that $D^{+} f$ and $D^{-} f$ exist for convex functions $f$, are leftcontinuous and rightcontinuous respectively, and satisfy $D^{-} f(x) \leq D^{+} f(x) \leq$ $D^{-} f(y)$ for $x<y$ ([38], 3.6.19).

Denote by $\mathcal{A}$ the class of admissible controls. It consists of all processes $C:=\left(C^{U},-C^{L}\right)^{\top}$, where the components $C^{U}, C^{L}$ are $\left\{\mathcal{F}_{t}\right\}$-adapted, its paths are a.s. left continuous with right hand limits (LCRL), increasing; further they satisfy $\|C\|_{[\mathbf{1}, \bar{l}]}^{2}<\infty$.

Standard data $(b, \sigma, C)$ for the forward and $(h, g, a, C)$ for the backward equation in (2.2) below are defined as follows:

- $b \in L_{\mathcal{F}}^{2}\left(0, T ; W^{1, \infty}(\mathbb{R}, \mathbb{R})\right)$ and $\sigma \in L_{\mathcal{F}}^{2}\left(0, T ; W^{1, \infty}\left(\mathbb{R}, \mathbb{R}^{d}\right)\right)$.

- $C \in \mathcal{A}$ and $a=\left(a^{U}, a^{L}\right): \bar{\imath} \times \Omega \rightarrow \mathbb{R}^{2}$ progressively measurable such that for all $C^{\prime} \in \mathcal{A}$ the process $\int_{[0, t)} a_{s}^{\top} d C_{s}^{\prime}$ is in $L_{\mathcal{F}}^{2}(0, T ; \mathbb{R})$ and has LCRL paths $\boldsymbol{P}$-a.s.

- $g \in L_{\mathcal{F}}^{2}\left(0, T ; W^{1, \infty}\left(\mathbb{R} \times \mathbb{R} \times \mathbb{R}^{d}, \mathbb{R}\right)\right)$ and $h \in L_{\mathcal{F}_{T}}^{2}\left(\Omega ; W^{1, \infty}(\mathbb{R}, \mathbb{R})\right)$.

For $\left(t_{0}, x\right) \in \bar{\imath} \times \mathbb{R}$ and standard data $(b, \sigma, C, h, g, a)$ we consider the controlled FBSDE

$$
\begin{aligned}
d X_{u} & =b\left(u, X_{u}\right) d u-\mathbf{1}^{\top} d C_{u}+\sigma\left(u, X_{u}\right) d W_{u}, \\
d Y_{u} & =-g\left(u, X_{u}, Y_{u}, Z_{u}\right) d u-a_{u}^{\top} d C_{u}+Z_{u}^{\top} d W_{u}, \\
X_{t_{0}} & =x \\
Y_{T} & =\xi=h\left(X_{T}\right) .
\end{aligned}
$$

We write $\mathbf{1}^{\top} d C=(1,1) d C=d C^{U}-d C^{L}$. If no confusion is possible we abbreviate this by $d C$, and further abusing notation we may write $C$ instead of $C^{U}-C^{L}$ from time to time. As we will show below (2.2) has a unique solution $(X, Y, Z) \in L_{\mathcal{F}}^{2}\left(0, T ; \mathbb{R} \times \mathbb{R} \times \mathbb{R}^{d}\right)$. Dependence on data and parameters $t_{0}, x, b, \sigma, C, \xi, h, a, g$ may be expressed by a superscript.

Observe that forward and backward equation are decoupled in the sense that $X$ does not depend on $Y$. From time to time we may consider the backward equation without reference to a particular forward process. In these cases we will omit dependence of $g$ on $x$ and write $\xi$ for the terminal condition, with suitable adaption of the meaning of standard data. 
Note. In a more general setting $a$ will depend also on $(x, y)$. Without having defined precisely the notion of a solution for (2.2) we will present a few statements in this more general situation. We then tacitly assume that the formulation of standard data with respect to $a$ is such that a unique adapted, square integrable solution exists.

\subsection{Definition of control problem and Dynkin game}

Control problem For $t_{0} \in \bar{\imath}, x \in \mathbb{R}$ and $C \in \mathcal{A}$ the solution to the FBSDE (2.2) with data $(b, \sigma, C, h, a, g)$ is denoted by $\left(X_{t}^{t_{0}, x, C}, Y_{t}^{t_{0}, x, C}, Z_{t}^{t_{0}, x, C}\right)$. Then $Y_{t}^{t_{0}, x, C}$ is called the cost associated with control $C$. The value of the control problem with dynamics $(b, \sigma)$ and cost structure $(h, g, a)$ is the process defined as

$$
V_{t}\left(t_{0}, x\right):=\operatorname{essinf}_{C \in \mathcal{A}} Y_{t}^{t_{0}, x, C}
$$

$V_{t}\left(t_{0}, x\right)<\infty$ follows from $Y_{t}^{t_{0}, x, 0}<\infty$. In addition we assume $-\infty<$ $V_{t}\left(t_{0}, x\right)<\infty$ for all $t, t_{0}, x$.

Definition 2.2. The control problem comprises determining the value process $V$ and finding an optimal control $C^{\star} \in \mathcal{A}$ with the property

$$
\forall t \in \bar{\imath} \quad V_{t}\left(t_{0}, x\right)=Y_{t}^{t_{0}, x, C^{\star}} \quad[\boldsymbol{P}] .
$$

Remark 2.3. Additional restrictions on starting values and admissible controls could be imposed. For example, in problems with a so called generalized finite fuel condition the starting values are from an interval $J \subset \mathbb{R}$, and admissible controls $\mathcal{A}_{J}$ are such that the controlled forward process $X^{t_{0}, x, C}$ stays in the interval $\left[X^{t_{0}, \min J, 0}, X^{t_{0}, \max J, 0}\right]$. Under a strict finite fuel condition, a bound is imposed on $\left|C_{T}\right|$. See [34], [12] for a discussion of these types of problems.

Associated stochastic game of optimal stopping We consider a twoplayer stochastic game of optimal stopping or Dynkin game related with above control problem. $\mathcal{T}_{t_{0}}$ denotes the class of $\mathcal{F}_{t}$-stopping times with values a.s. in $\left[t_{0}, T\right]$. Let $X^{t_{0}, x, 0,0}$ the uncontrolled solution of the FSDE in (2.2) with data $(b, \sigma, 0), \Gamma^{x}$ a deflator process defined in (2.34) below, and $\sigma, \tau \in \mathcal{T}_{t_{0}}$. Assume for a while that $h$ and $g$ are partially differentiable with respect to $(x, y, z)$ and $g$ is linear in $z$ (hence $D g$ independent of $z$ ). Define the payoff $R_{t}^{t_{0}, x}(\sigma, \tau)=R_{t}$, where $(R, Q)$ is the solution of the BSDE

$$
\begin{aligned}
d R_{u} & =-\left\langle D g\left(t, X_{t}^{t_{0}, x, 0,0}, V_{t}\left(t_{0}, x\right)\right),\left(\Gamma_{t}^{x}, R_{t}, Q_{t}\right)\right\rangle \boldsymbol{\chi}_{t \leq \sigma \wedge \tau} d t+Q_{t}^{\top} d W_{t}, \\
R_{T} & =\left(h_{x}\left(X_{T}^{t_{0}, x, 0,0}\right) \boldsymbol{\chi}_{\sigma \wedge \tau=T}+a_{\tau}^{L} \boldsymbol{\chi}_{\tau<\sigma}+a_{\sigma<T}^{U} \boldsymbol{\chi}_{\sigma<\tau}\right) \Gamma_{\sigma<T}^{x} \Gamma_{\sigma \wedge \tau \wedge T} .
\end{aligned}
$$

and consider the upper and lower values

$$
\begin{aligned}
& u_{t}^{+}\left(t_{0}, x\right):=\operatorname{essinf}_{\sigma \in \mathcal{T}_{t}} \operatorname{ess}_{\sup _{\tau \in \mathcal{T}_{t}} R_{t}^{t_{0}, x}(\sigma, \tau)} \\
& u_{t}^{-}\left(t_{0}, x\right):=\operatorname{ess}_{\sup _{\tau \in \mathcal{T}_{t}}} \operatorname{essinf}_{\sigma \in \mathcal{T}_{t}} R_{t}^{t_{0}, x}(\sigma, \tau)
\end{aligned}
$$

We assume $-\infty<u_{t}^{-}\left(t_{0}, x\right)$ for all $\left(t, t_{0}, x\right)$.

Remark 2.4. Observe that, by the definition of $u^{+}$and $u^{-}, u^{-} \leq u^{+}$holds $\boldsymbol{P}$-a.s. 
The value process $u$ of the Dynkin game is the process defined as the solution of the Isaac's equation

$$
u_{t}\left(t_{0}, x\right):=u_{t}^{+}\left(t_{0}, x\right)=u_{t}^{-}\left(t_{0}, x\right)
$$

if this equality holds.

This has an interpretation as a game for two players I, II: I pays II at rate $\langle D g, \cdot\rangle$ as long as the game continues, and $h_{x} \Gamma_{T}$ upon reaching the time horizon $T$. Both players I and II have the possibility to force early termination of the game at $\sigma$ and $\tau$ respectively. On early termination, I pays II $a_{\sigma}^{U} \Gamma_{\sigma}$ or $a_{\tau}^{L} \Gamma_{\tau}$, depending on whether I or II stopped the game. So I seeks to minimize $R^{x, t_{0}}(\sigma, \tau)$ by choice of $\sigma$, whereas II wishes to maximize the payoff by choice of $\tau$. Intuitively speaking, a solution consists of a pair of stopping times such that both players have no incentive to deviate from their strategy. The optimization of the opponent is anticipated and the right of choosing ones strategy first gives no advantage, i.e. has no additional value, which is the meaning of the Isaac's equation (2.7).

Definition 2.5. The associated stochastic game of optimal stopping or Dynkin game consists of determining the upper and lower values $u^{+}$and $u^{-}$and the value process $u$ as solution to the Isaac's equation, and of finding a saddle point $\left(\sigma^{\star}, \tau^{\star}\right)$, i.e. optimal stopping times such that

$\forall t \in \bar{\imath}$

$$
\begin{aligned}
& u_{t}\left(t_{0}, x\right)=R_{t}^{t_{0}, x}\left(\sigma^{\star}, \tau^{\star}\right) \\
& =\operatorname{essinf}_{\sigma \in \mathcal{T}_{t_{0}}} R_{t}^{t_{0}, x}\left(\sigma, \tau^{\star}\right)=\operatorname{ess}_{\sup _{\tau \in \mathcal{T}_{t_{0}}}} R_{t}^{t_{0}, x}\left(\sigma^{\star}, \tau\right)
\end{aligned}
$$

For simplicity we assume $t_{0}=0$ from now on and hence omit dependence on $t_{0}$ in the notation.

\subsection{Existence and uniqueness of solutions}

Let $(f, C, \sigma, h, g, a)$ standard data of the controlled FBSDE. We use a transformation that makes it possible to apply standard results on existence and uniqueness of solutions to FSDE and BSDE as can be found in e.g. [38], [40], [51] [30], [24], [58], [42].

Define dynamics $\tilde{b} \in L_{\mathcal{F}}^{2}\left(0, T ; W^{1, \infty}(\mathbb{R}, \mathbb{R})\right), \tilde{\sigma} \in L_{\mathcal{F}}^{2}\left(0, T ; W^{1, \infty}\left(\mathbb{R}, \mathbb{R}^{d}\right)\right)$, and cost structure $\tilde{g} \in L_{\mathcal{F}}^{2}\left(0, T ; W^{1, \infty}\left(\mathbb{R} \times \mathbb{R} \times \mathbb{R}^{d}, \mathbb{R}\right)\right), \tilde{h} \in L_{\mathcal{F}_{T}}^{2}\left(\Omega ; W^{1, \infty}(\mathbb{R}, \mathbb{R})\right)$ by

$$
\begin{aligned}
\tilde{b}(t, x) & :=b\left(t, x-C_{t}\right), & \tilde{\sigma}(t, x) & :=\sigma\left(t, x-C_{t}\right), \\
\tilde{g}(t, x, y, z) & :=\tilde{g}\left(t, x, y-\int_{[0, t)} a_{s}^{\top} d C_{s}, z\right), & \tilde{h}(x) & :=h(x)+\int_{[0, T)} a_{s}^{\top} d C_{s} .
\end{aligned}
$$

Here we have omitted dependence on $\omega$. Let $\tilde{X}$ the unique solution of

$$
d \tilde{X}_{t}=\tilde{b}\left(t, \tilde{X}_{t}\right) d t+\tilde{\sigma}\left(t, \tilde{X}_{t}\right)^{\top} d W_{t}, \quad \tilde{X}_{0}=x_{0} .
$$

This implies that $X_{t}:=\tilde{X}_{t}-C_{t}$ solves the forward SDE in (2.2) with data $(b, C, \sigma)$. Similarly, let $(\tilde{Y}, \tilde{Z})$ the unique solution of the BSDE

$$
d \tilde{Y}_{t}=\tilde{g}\left(t, X_{t}, \tilde{Y}_{t}, \tilde{Z}_{t}\right) d t+\tilde{Z}_{t}^{\top} d W_{t}, \quad \tilde{Y}_{T}=\tilde{h}\left(X_{t}\right) .
$$

Then $\left(Y_{t}, Z_{t}\right):=\left(\tilde{Y}_{t}-\int_{[0, t)} a_{s}^{\top} d C_{s}, \tilde{Z}_{t}\right)$ solves the backward SDE in (2.2) with data $(h, g, a, C)$. 


\subsection{A priori estimate and comparison theorem for forward equation}

For an estimate of $E\left[\left|X_{t}\right|^{p}\right]$ in case $C=0$ see [25], App. D, or [40], Ch. 2.5, lemma 2, cor. 5,6 .

Lemma 2.6. (A Priori Estimates) Let, for $i \in\{1,2\},\left(b^{i}, \sigma^{i}, C^{i}\right)$ standard data, $x^{i} \in \mathbb{R}$ and $X^{i}$ the corresponding solutions of the FSDE. Define

$$
\begin{aligned}
& \boldsymbol{\delta} X_{t}:=X_{t}^{1}-X_{t}^{2} \quad \delta x:=x^{1}-x^{2} \quad \delta C_{t}=C_{t}^{1}-C_{t}^{2} \\
& \boldsymbol{\delta} b_{t}:=b^{1}\left(t, X_{t}^{2}\right)-b^{2}\left(t, X_{t}^{2}\right) \quad \boldsymbol{\delta} \sigma_{t}:=\sigma^{1}\left(t, X_{t}^{2}\right)-\sigma^{2}\left(t, X_{t}^{2}\right)
\end{aligned}
$$

Then there is a constant $K_{4}$ depending only on $T$ and $L$ such that

$$
E\left[\sup _{\bar{\imath}}\left|\boldsymbol{\delta} X_{t}\right|^{2}\right] \leq K_{4}\left(|\boldsymbol{\delta} x|^{2}+\|\boldsymbol{\delta} b\|_{2}^{2}+\|\boldsymbol{\delta} \sigma\|_{2}^{2}+E\left[|\boldsymbol{\delta} C|_{T}^{2}\right) .\right.
$$

Proof. As $\boldsymbol{\delta} X$ can be written as $\boldsymbol{\delta} X_{t}=\boldsymbol{\delta} x+B_{t}-\boldsymbol{\delta} C_{t}+M_{t}$ with suitable $B, M$ we have the estimate

$$
\begin{aligned}
& \sup _{0 \leq s \leq t}\left(\boldsymbol{\delta} X_{s}\right)^{2} \leq 4|\boldsymbol{\delta} x|^{2}+4 \sup _{0 \leq s \leq t}\left(\int_{0}^{s} b^{1}\left(u, X_{u}^{1}\right)-b^{2}\left(u, X_{u}^{2}\right) d u\right)^{2} \\
& \quad+4 \sup _{0 \leq s \leq t}\left(-\delta C_{s}\right)^{2}+4 \sup _{0 \leq s \leq t}\left(\int_{0}^{s}\left(\sigma^{1}\left(u, X_{u}^{1}\right)-\sigma^{2}\left(u, X_{u}^{2}\right)\right)^{\top} d W_{u}\right)^{2}
\end{aligned}
$$

Now from the Hölder inequality $\|f g\| \leq\|f\|_{p}\|g\|_{q}$ (for $p=q=2$ and $f=1$, $\left.g=b^{1}-b^{2}\right)$ and Burkholder-Davis-Gundy inequality ([38], Theorem 3.3.28) it follows that

$$
\begin{aligned}
E\left[\sup _{0 \leq s \leq t}\left(\boldsymbol{\delta} X_{s}\right)^{2}\right] \leq & 4|\boldsymbol{\delta} x|^{2}+4 t E\left[\int_{0}^{t}\left(b^{1}\left(s, X_{s}^{1}\right)-b^{2}\left(s, X_{s}^{2}\right)\right)^{2} d s\right] \\
& +4 E\left[|\boldsymbol{\delta} C|_{t}^{2}\right]+4 K_{1} E\left[\int_{0}^{t}\left|\sigma^{1}\left(s, X_{s}^{1}\right)-\sigma^{2}\left(s, X_{s}^{2}\right)\right|^{2} d s\right] \\
\leq & 4|\boldsymbol{\delta} x|^{2}+4 t \int_{0}^{t} 2 L^{2} E\left[\left(\boldsymbol{\delta} X_{s}\right)^{2}\right]+2 E\left[\left(\boldsymbol{\delta} b_{s}\right)^{2}\right] d s \\
& +4 E\left[|\boldsymbol{\delta} C|_{t}^{2}\right]+4 K_{1} \int_{0}^{t} 2 L^{2} E\left[\left(\boldsymbol{\delta} X_{s}\right)^{2}\right]+2 E\left[\left(\boldsymbol{\delta} \sigma_{s}\right)^{2}\right] d s \\
\leq & 4|\boldsymbol{\delta} x|^{2}+8 t\|\boldsymbol{\delta} b\|_{2}^{2}+8 K_{1}\|\boldsymbol{\delta} \sigma\|_{2}^{2}+4 E\left[|\boldsymbol{\delta} C|_{t}^{2}\right] \\
& +8 L^{2}\left(t+K_{1}\right) \int_{0}^{t} E\left[\left|\boldsymbol{\delta} X_{s}\right|^{2}\right] d s
\end{aligned}
$$


On the other hand, if we apply Itô's formula to $|\boldsymbol{\delta} X|^{2}$ and take expectation, it follows that

$$
\begin{array}{r}
E\left[\left|\boldsymbol{\delta} X_{t}\right|^{2}\right]=|\boldsymbol{\delta} x|^{2}+2 E\left[\int_{0}^{t} \boldsymbol{\delta} X_{s}\left(b^{1}\left(s, X_{s}^{1}\right)-\left(b^{1}\left(s, X_{s}^{2}\right)\right)+\boldsymbol{\delta} X_{s} \boldsymbol{\delta} b_{s} d s\right]\right. \\
-2 E\left[\int_{0}^{t} \boldsymbol{\delta} X_{s} d\left(\boldsymbol{\delta} C_{s}\right)\right]+E\left[\int_{0}^{t}\left|\sigma^{1}\left(s, X_{s}^{1}\right)-\sigma^{1}\left(s, X_{s}^{2}\right)+\boldsymbol{\delta} \sigma_{s}\right|^{2} d s\right] \\
\leq|\boldsymbol{\delta} x|^{2}+\|\boldsymbol{\delta} b\|_{2}^{2}+2\|\boldsymbol{\delta} \sigma\|_{2}^{2}+E\left[\sup _{0 \leq s \leq t}\left|\boldsymbol{\delta} X_{s}\right|^{2}\right]+E\left[|\boldsymbol{\delta} C|_{t}^{2}\right] \\
+\int_{0}^{t}\left(2 L+2 L^{2}+1\right) E\left[\left|\boldsymbol{\delta} X_{s}\right|^{2}\right] d s
\end{array}
$$

Now insert (2.11) in (2.12). At this point observe that $t \mapsto E\left[\left|\boldsymbol{\delta} X_{t}\right|^{2}\right]$ is continuous a.e. on $\bar{\imath}$ by Lemma 2.7 below. Hence an application of the Gronwall inequality (cf. [38], problem 5.2 .7 or [11] p. 12.) shows that the following holds for Lebesgue-almost all $t \in \bar{\imath}$ :

$$
E\left[\left|\boldsymbol{\delta} X_{t}\right|^{2}\right] \leq K_{2}\left(|\boldsymbol{\delta} x|^{2}+\|\boldsymbol{\delta} b\|_{2}^{2}+\|\boldsymbol{\delta} \sigma\|_{2}^{2}+E\left[|\boldsymbol{\delta} C|_{T}^{2}\right]\right) e^{2 K_{3} t} .
$$

Here $K_{3}=8 L^{2}\left(T+K_{1}\right)+2 L+2 L^{2}+1$, and both $K_{2}$ and $K_{3}$ depend only on $L$ and $T$. As the right hand side of (2.13) is increasing in $t$ we conclude that the estimate therein holds a.e. for $\sup _{s<t} E\left[\left|\boldsymbol{\delta} X_{t}\right|^{2}\right]$ as well. Hence (2.13) holds for all $t \in \bar{\imath}$ as the right hand side is continuous in $t$.

Now (2.9) can be deduced from (2.11) and (2.13).

Lemma 2.7. The maps $t \mapsto E\left[\left|\boldsymbol{\delta} X_{t}\right|^{2}\right]$ and $t \mapsto E\left[|C|_{t}\right]$ are continuous a.e. in $\bar{\imath}$.

Proof. By (2.12) and definition of $|C|$ the set discontinuities of both maps is a subset of the set of discontinuities of the maps $t \mapsto E\left[C_{t}^{U}\right]$ and $t \mapsto E\left[C_{t}^{U}\right]$. Hence it suffices to show that the latter have countably many discontinuities in $\bar{\imath}$. But this is obvious as for an increasing process $\gamma_{t}$ with $E\left[\gamma_{T}\right]<\infty$ the map $t \mapsto E\left[\gamma_{t}\right]$ is increasing and bounded, hence has its set of discontinuities is countable.

We continue to use the notation of lemma 2.6.

Theorem 2.8. (Comparison Theorem) Let, for $i \in\{1,2\},\left(b^{i}, \sigma^{i}, C^{i}\right)$ standard data, $x^{i} \in \mathbb{R}$ and $X^{i}$ the corresponding solutions of the FSDE. Assume further that

$$
\begin{aligned}
& b^{1}(\omega, t, x) \geq b^{2}(\omega, t, x) \\
& -\mathbf{1}^{\top} d\left(\boldsymbol{\delta} C_{t}\right) \geq 0 \quad \text { (i.e. } \boldsymbol{\delta} C \text { decreasing) } \\
& \sigma^{1}(\omega, t, x)=\sigma^{2}(\omega, t, x) \\
& \boldsymbol{\delta} x \geq 0
\end{aligned}
$$

Then the difference $\boldsymbol{\delta} X_{t}$ is positive almost surely:

$$
X_{t}^{1} \geq X_{t}^{2} \quad \forall t \in \bar{\imath} \quad[\boldsymbol{P}]
$$

If $A:=\left\{\omega \mid X_{t}^{1}(\omega)=X_{t}^{2}(\omega)\right\}$ has positive probability, then

$$
x^{1}=x^{2}, \quad b^{1}(\omega, s, x)=b^{2}(\omega, s, x) \quad \forall s \in[0, t], \quad \forall \omega \in A
$$


Remark 2.9. We will see in the proof that instead of $b^{1} \geq b^{2}$ it is actually sufficient if one of the conditions

$$
b^{1}\left(\omega, t, X_{t}^{1}\right) \geq b^{2}\left(\omega, t, X_{t}^{1}\right), \quad b^{1}\left(\omega, t, X_{t}^{2}\right) \geq b^{2}\left(\omega, t, X_{t}^{2}\right)
$$

holds. If e.g. $b^{1}$ is independent of $x$ this reduces to $b^{1}(\omega, t) \geq b^{2}\left(\omega, t, X_{t}^{1}\right)$.

Note. The assumption on $\delta C$ is satisfied if both $C_{t}^{U, 2}-C_{t}^{U, 1}$ and $C_{t}^{L, 1}-C_{t}^{L, 2}$ are increasing, but usually there will be weaker conditions.

By applying theorem 2.8 to $X^{2}=0$ we deduce

Corollary 2.10. Let $(b, \sigma, C)$ standard data for the forward SDE satisfying $b \geq$ $0, \sigma(t, 0)=0$ and $C^{U}=0$, and let $x \geq 0$. Then the solution of the FSDE is nonnegative.

To prove theorem 2.8 we linearize the difference of solutions (idea from [24]). So we first state and prove a lemma on affine FSDE.

Lemma 2.11. Assume $(b, \sigma, C)$ are standard data of a FSDE, where $b, \sigma$ are affine, so we consider an equation of the form

$$
d X_{t}=\left(\beta_{t} X_{t}+\varphi_{t}\right) d t-\mathbf{1}^{\top} d C_{t}+\left(\varsigma_{t} X_{t}+\psi_{t}\right)^{\top} d W_{t}, \quad X_{0}=x
$$

with suitable progressively measurable processes $\beta, \varsigma, \varphi, \psi, \beta, \varsigma$ are bounded. Let $\Gamma_{s}^{t}$ defined by

$$
\Gamma_{s}^{t}=\exp \left(\int_{s}^{t} \beta_{u}-\frac{1}{2}\left|\varsigma_{u}\right|^{2} d u+\int_{s}^{t} \varsigma_{u}^{\top} d W_{u}\right)
$$

Then $X$ has the representation

$$
X_{t}=\Gamma_{0}^{t} x+\int_{0}^{t} \Gamma_{s}^{t} \varphi_{s}-\Gamma_{s}^{t}\left\langle\varsigma_{s}, \psi_{s}\right\rangle d s-\int_{0}^{t} \Gamma_{s}^{t} \mathbf{1}^{\top} d C_{s}+\int_{0}^{t} \Gamma_{s}^{t} \psi_{s}^{\top} d W_{s}
$$

In particular, if $x \geq 0, \varphi \geq 0, C$ decreasing and $\psi=0$ then $X_{t} \geq 0$ a.s. If $A:=\left\{\omega \mid X_{t}=0\right\}$ has positive probability, then $x=\varphi_{s}=C_{s}=0$ for $0 \leq s \leq t$ on $A$ P-a.s.

Proof. Let $\Gamma_{t}$ the solution of the homogeneous LSDE

$$
d \Gamma_{t}=-\Gamma_{t}\left(\left(\beta_{t}-\left|\varsigma_{t}\right|^{2}\right) d t+\varsigma_{t}^{\top} d W_{t}\right), \quad \Gamma_{0}=1
$$

Then $\Gamma_{t}$ has the representation (2.16), i.e. $\Gamma_{t}=\Gamma_{t}^{0}$, and $\Gamma_{s}^{t}=\left(\Gamma_{t}\right)^{-1} \Gamma_{s}$.

Now apply the Itô formula to $\Gamma_{t} X_{t}$

$$
\Gamma_{t} X_{t}-x=\int_{0}^{t} \Gamma_{s} \varphi_{s}-\Gamma_{s}\left\langle\varsigma_{s}, \psi_{s}\right\rangle d s-\int_{0}^{t} \Gamma_{s} \mathbf{1}^{\top} d C_{s}+\int_{0}^{t} \Gamma_{s} \psi_{s}^{\top} d W_{s}
$$

and deduce (2.17). If $\psi=0$ the second and last term drop out, and $X \geq 0$ follows from $\Gamma \geq 0$ and the conditions $x \geq 0, \varphi \geq 0$ and $C$ decreasing.

Proof of theorem 2.8. Define the bounded, progressively measurable processes

$$
\begin{aligned}
& \Delta_{x} b_{t}:=\frac{1}{X_{t}^{1}-X_{t}^{2}}\left(b^{1}\left(t, X_{t}^{1}\right)-b^{1}\left(t, X_{t}^{2}\right)\right) \chi_{X_{t}^{1} \neq X_{t}^{2}} \\
& \Delta_{x} \sigma_{t}:=\frac{1}{X_{t}^{1}-X_{t}^{2}}\left(\sigma^{1}\left(t, X_{t}^{1}\right)-\sigma^{2}\left(t, X_{t}^{2}\right)\right) \chi_{X_{t}^{1} \neq X_{t}^{2}}
\end{aligned}
$$


and write the SDE for $\delta X$ in the form as in (2.15)

$$
d\left(\boldsymbol{\delta} X_{t}\right)=\left(\Delta_{x} b_{t} \boldsymbol{\delta} X_{t}+\boldsymbol{\delta} b_{t}\right) d t-\mathbf{1}^{\top} d\left(\boldsymbol{\delta} C_{t}\right)+\boldsymbol{\delta} X_{t}\left(\Delta_{x} \sigma_{t}\right)^{\top} d W_{t}, \quad \boldsymbol{\delta} X_{0}=\boldsymbol{\delta} x .
$$

Here $\boldsymbol{\delta} b \geq 0$. Recall that $\Delta_{x} \sigma$ is bounded as $\sigma^{1}=\sigma^{2}$ by assumption. Now apply lemma 2.11 to conclude.

Remark 2.12. It is obvious from the proof that the conclusion of theorem 2.8 would remain true if instead of $\sigma^{1}=\sigma^{2}$ we knew that $\Delta_{x} \sigma_{t}$ is bounded. However if $\sigma^{1} \neq \sigma^{2}$ and $X^{i}$ are diffusions this is not true.

\subsection{A priori estimates and Comparison Theorem for BSDE}

Lemma 2.13. (A Priori Estimates) Let, for $i \in\{1,2\},\left(g^{i}, \xi^{i}, a^{i}, C^{i}\right)$ standard data and $\left(Y^{i}, Z^{i}\right)$ the corresponding solutions of the BSDE (2.2). Define

$$
\begin{array}{rlrlrl}
\delta Y_{t} & :=Y_{t}^{1}-Y_{t}^{2} & \delta Z_{t}:=Z_{t}^{1}-Z_{t}^{2} & \boldsymbol{\delta} \xi:=\xi^{1}-\xi^{2} \\
\boldsymbol{\delta} g_{t}:=g^{1}\left(t, Y_{t}^{2}, Z_{t}^{2}\right)-g^{2}\left(t, Y_{t}^{2}, Z_{t}^{2}\right) & \boldsymbol{\delta} a_{t}:=a_{t}^{1} a_{t}^{2} & \delta C_{t}:=C_{t}^{1}-C_{t}^{2}
\end{array}
$$

Then there are constants depending only on $L$ and $T$ such that for all $t \leq T$

$$
E\left[\int_{t}^{T}\left|\boldsymbol{\delta} Z_{s}\right|^{2} d s\right] \leq K_{2}\left(\|\boldsymbol{\delta} \xi\|_{2}^{2}+\|\boldsymbol{\delta} g\|_{2}^{2}+\|\boldsymbol{\delta} a\|_{\left[C^{1}, \bar{l}\right]}^{2}+|\boldsymbol{\delta} C|_{\left[a^{2}, \bar{l}\right]}^{2}\right)\left(1+e^{K_{3}(T-t)}\right)
$$

and the running maximum of differences satisfies

$$
E\left[\sup _{\bar{\imath}}\left|\boldsymbol{\delta} Y_{t}\right|^{2}\right] \leq K_{4}\left(\|\boldsymbol{\delta} \xi\|_{2}^{2}+\|\boldsymbol{\delta} g\|_{2}^{2}+\|\boldsymbol{\delta} a\|_{\left[C^{1}, \bar{l}\right]}^{2}+|\boldsymbol{\delta} C|_{\left[a^{2}, \bar{l}\right.}^{2}\right] .
$$

Proof. First of all, write $\delta Y$ in the integral form

$$
\begin{aligned}
\delta Y_{t}= & \delta \xi+\int_{t}^{T} g^{1}\left(s, Y_{s}^{1}, Z_{s}^{1}\right)-g^{2}\left(s, Y_{s}^{2}, Z_{s}^{2}\right) d s \\
& +\left(\int_{[t, T)} a^{1}(s)^{\top} d C_{s}^{1}-\int_{[t, T)} a^{2}(s)^{\top} d C_{s}^{2}\right)+\int_{t}^{T} \delta Z_{s}^{\top} d W_{s}
\end{aligned}
$$

Take squares and sup on both sides. Here and in the following we make excessive use of the "binomial inequality" $(a+b)^{2} \leq 2 a^{2}+2 b^{2}$. Apply the Hölder inequality $\|f g\| \leq\|f\|_{p}\|g\|_{q}$ with $f=1, g=g^{1}-\bar{g}^{2}$ and $p=q=\frac{1}{2}$ and rewrite the terms in $C$ and $g$ to get

$$
\begin{aligned}
& \sup _{t \leq s \leq T}\left|\boldsymbol{\delta} Y_{s}\right|^{2} \leq 4|\boldsymbol{\delta} \xi|^{2}+4(T-t) \int_{t}^{T}\left(g^{1}\left(s, Y_{s}^{1}, Z_{s}^{1}\right)-g^{1}\left(s, Y_{s}^{2}, Z_{s}^{2}\right)+\boldsymbol{\delta} g_{s}\right)^{2} d s \\
& +4\left(\int_{\bar{\imath}} \boldsymbol{\delta} a_{s}^{\top} d C_{s}^{1}+\int_{\bar{\imath}} a^{2}(s)^{\top} d\left(\boldsymbol{\delta} C_{s}\right)\right)^{2}+\sup _{t \leq s \leq T}\left(\int_{s}^{T}\left(\boldsymbol{\delta} Z_{s}\right)^{\top} d W_{s}\right)^{2} \cdot \quad(2.22)
\end{aligned}
$$

Now take expectations, use Burkholder-Davis-Gundy inequality and Lipschitz continuity of $g$ and rearrange terms:

$$
\begin{aligned}
& E\left[\sup _{t \leq s \leq T}\left|\boldsymbol{\delta} Y_{s}\right|^{2}\right] \leq 4\|\boldsymbol{\delta} \xi\|_{2}^{2}+8(T-t)\|\boldsymbol{\delta} g\|_{2}^{2}+8 L^{2}(T-t) \int_{t}^{T} E\left[\left|\boldsymbol{\delta} Y_{s}\right|^{2}\right] d s \\
& +\left(8 L^{2}(T-t)+4 K_{1}\right) E\left[\int_{t}^{T}\left|\boldsymbol{\delta} Z_{s}\right|^{2} d s\right]+16\|\boldsymbol{\delta} a\|_{\left[C^{1}, \bar{l}\right]}^{2}+16|\boldsymbol{\delta} C|_{\left[a^{2}, \bar{l}\right]}^{2}, \quad
\end{aligned}
$$


where $K_{1}$ is the universal constant of the Burkholder-Davis-Gundy inequality (cf. [38] Theorem 3.3.28).

If we apply Itô's formula to $\left|\delta Y_{t}\right|^{2}$ and take expectations, recalling that $\delta Y$ is square integrable, then

$$
\begin{aligned}
& E\left[\left|\boldsymbol{\delta} Y_{t}\right|^{2}\right]+E\left[\int_{t}^{T}\left|\boldsymbol{\delta} Z_{s}\right|^{2} d s\right]=\|\boldsymbol{\delta} \xi\|_{2}^{2} \\
&+ 2 E\left[\int_{t}^{T} \boldsymbol{\delta} Y_{s}\left(g^{1}\left(s, Y_{s}^{1}, Z_{s}^{1}\right)-g^{1}\left(s, Y_{s}^{2}, Z_{s}^{2}\right)\right)+\boldsymbol{\delta} Y_{s} \boldsymbol{\delta} g_{s} d s\right] \\
&+ 2 E\left[\int_{[t, T)} \boldsymbol{\delta} Y_{s}\left(\boldsymbol{\delta} a_{s}\right)^{\top} d C_{s}^{1}\right]+2 E\left[\int_{[t, T)} \boldsymbol{\delta} Y_{s} a^{2}(s)^{\top} d\left(\boldsymbol{\delta} C_{s}\right)\right] \\
& \leq\|\boldsymbol{\delta} \xi\|_{2}^{2}+\|\boldsymbol{\delta} g\|_{2}^{2}+\frac{1}{2} E\left[\int_{t}^{T}\left|\boldsymbol{\delta} Z_{s}\right|^{2} d s\right]+\left(2 L+2 L^{2}+1\right) \int_{t}^{T} E\left[\left|\boldsymbol{\delta} Y_{s}\right|^{2}\right] d s \\
& \quad+4 \epsilon E\left[\sup _{t \leq s \leq T}\left|\boldsymbol{\delta} Y_{s}\right|^{2}\right]+\frac{1}{\epsilon}\left\|\boldsymbol{\delta} a_{s}\right\|_{\left[C^{1}, \bar{l}\right]}^{2}+\frac{1}{\epsilon}|\boldsymbol{\delta} C|_{\left[a^{2}, \bar{l}\right]}^{2} . \quad(2.24)
\end{aligned}
$$

Now specify $\epsilon:=\left(16\left(8 L^{2}(T-t)+4 K_{1}\right)\right)^{-1}>0$, apply $(2.23)$ in (2.24) and rearrange terms to arrive at

$$
\begin{array}{r}
E\left[\left|\boldsymbol{\delta} Y_{t}\right|^{2}\right]+\frac{1}{4} E\left[\int_{t}^{T}\left|\boldsymbol{\delta} Z_{s}\right|^{2} d s\right] \leq\left(2 L+2 L^{2}+1+4 \epsilon 8 L^{2}(T-t)\right) \int_{t}^{T} E\left[\left|\boldsymbol{\delta} Y_{s}\right|^{2}\right] d s \\
+K_{2}\left(\|\boldsymbol{\delta} \xi\|_{2}^{2}+\|\boldsymbol{\delta} g\|_{2}^{2}+\left\|\boldsymbol{\delta} a_{s}\right\|_{\left[C^{1}, \bar{\imath}\right]}^{2}+|\boldsymbol{\delta} C|_{\left[a^{2}, \bar{l}\right.}^{2}\right) .
\end{array}
$$

Using Gronwall's inequality we can deduce that for almost all $t \in \bar{\imath}$ :

$$
E\left[\left|\boldsymbol{\delta} Y_{t}\right|^{2}\right] \leq K_{2}\left(\|\boldsymbol{\delta} \xi\|_{2}^{2}+\|\boldsymbol{\delta} g\|_{2}^{2}+\|\boldsymbol{\delta} a\|_{\left[C^{1}, \bar{l}\right]}^{2}+|\boldsymbol{\delta} C|_{\left[a^{2}, \bar{l}\right]}^{2}\right) e^{K_{3}(T-t)} .
$$

An argument analogous to that in the proof of (2.13) and lemma 2.7 shows that (2.26) holds for all $t \in \bar{\imath}$.

Now (2.20) follows from (2.23) and (2.26). Combining (2.26) with (2.25) yields (2.19).

Similarly to the forward situation we now prove a Comparison Theorem for the backward equation in (2.2).

Theorem 2.14. (Comparison Theorem) Let, for $i \in\{1,2\},\left(g^{i}, \xi^{i}, a^{i}, C^{i}\right)$ standard data and $\left(Y^{i}, Z^{i}\right)$ the corresponding solutions of the BSDE. $a^{i}$ may depend on $Y$. Assume further that $\boldsymbol{P}$-a.s.

$$
\begin{aligned}
g^{1}(t, y, z)-g^{2}(t, y, z) & \geq 0 & \xi^{1}-\xi^{2} \geq 0 \\
a^{1}(t, y)^{\top} d\left(C_{t}^{1}-C_{t}^{2}\right) & \geq 0 & \left(a^{1}(t, y)-a^{2}(t, y)\right)^{\top} d C_{t}^{2} \geq 0
\end{aligned}
$$

Then the difference $\delta Y_{t}:=Y_{t}^{1}-Y_{t}^{2}$ is nonnegative almost surely. Let $A:=$ $\left\{\omega \mid Y_{t_{0}}^{1}(\omega)=Y_{t_{0}}^{2}(\omega)\right\}$. If $\boldsymbol{P}(A)>0$, then equality holds in (2.27) for $t_{0} \leq t \leq T$ and $\omega \in A \boldsymbol{P}$-a.s.

Remark 2.15. We will see in the proof that, instead of the first inequality in (2.27a), it is sufficient if one of the following conditions

$$
g^{1}\left(t, Y_{t}^{1}, Z_{t}^{1}\right)-g^{2}\left(t, Y_{t}^{1}, Z_{t}^{1}\right) \geq 0, \quad g^{1}\left(t, Y_{t}^{2}, Z_{t}^{2}\right)-g^{2}\left(t, Y_{t}^{2}, Z_{t}^{2}\right) \geq 0
$$


holds. This will prove useful in situations where we make use of the convexity of the driver $g(t, x, y, z)$.

In situations where e.g. $g^{1}$ does not depend on $\left(Y^{1}, Z^{1}\right)$ we conclude that $g_{t}^{1} \geq g^{2}\left(t, Y_{t}^{1}, Z_{t}^{1}\right)$ is also sufficient.

Remark 2.16. Instead of (2.27b) it is actually sufficient if we have

$$
\int_{[0, t)} a^{1}(s, y)^{\top} d C_{s}^{1}-\int_{[0, t)} a^{2}(s, y)^{\top} d C_{s}^{2} \quad \text { is increasing in } t .
$$

Hence the theorem remains true if $(2.27 \mathrm{~b})$ is replaced by $(2.27 \mathrm{~d})$ or

$$
a^{2}(t, y)^{\top} d\left(C_{t}^{1}-C_{t}^{2}\right) \geq 0, \quad\left(a^{1}(t, y)-a^{2}(t, y)\right)^{\top} d C_{t}^{1} \geq 0 .
$$

Remark 2.17. Often we can use properties of $Y^{i}$ when verifying (2.27a), e.g. $Y^{i} \geq 0$ because then we can restrict the estimate to $y \in \mathbb{R}_{\geq 0}$. On the contrary, we usually know very little about the processes $Z^{i}$. Hence, if one of the drivers is linear in $z,(2.27 \mathrm{a})$ in effect requires that the partial derivatives $g_{z}^{1}, g_{z}^{2}$ agree. This will force us to assume that $g_{z}$ is independent of $x, y, z$, as in the associated Dynkin game the driver takes the form $\left\langle\left(g_{x}, g_{y}, g_{z}\right),(\Gamma, R, Q)\right\rangle$.

Again we use a representation for solutions of linear BSDE:

Lemma 2.18. Let $(g, \xi, a, C)$ standard data of a linear BSDE and $(Y, Z)$ its solution, where the BSDE takes the form

$$
d Y_{t}=-\left(\gamma_{t}^{y} Y_{t}+\gamma_{t}^{z} Z_{t}+\varphi_{t}\right) d t-\left(Y_{t} \alpha_{t}^{y}\right)^{\top} d C_{t}-\psi_{t}^{\top} d \tilde{C}_{t}+Z_{t}^{\top} d W_{t}, \quad Y_{T}=\xi
$$

for certain progressively measurable processes $\gamma^{y}, \gamma^{z}, \alpha^{y}, \varphi, \psi, C, \tilde{C}$, where $\gamma^{y}$, $\gamma^{z}, \alpha^{y}$ are bounded and $C, \tilde{C}$ have bounded variation.

Let $\Gamma_{s}^{t}$ defined by

$$
\Gamma_{s}^{t}=\exp \left(\int_{s}^{t} \gamma_{u}^{y}-\frac{1}{2}\left|\gamma_{u}^{z}\right|^{2} d u+\int_{s}^{t} \alpha_{u}^{y \top} d C_{u}+\int_{s}^{t} \gamma_{u}^{z^{\top}} d W_{u}\right)
$$

Then $Y$ has the representation

$$
Y_{t}=E\left[\Gamma_{t}^{T} \xi+\int_{t}^{T} \Gamma_{t}^{s} \varphi_{s} d s+\int_{t}^{T} \Gamma_{t}^{s} \psi_{s}^{\top} d \tilde{C}_{s} \mid \mathcal{F}_{t}\right]
$$

In particular, if $\xi \geq 0, \varphi_{t} \geq 0$ and $\int_{[0, t)} \psi_{s}^{\top} d \tilde{C}_{s}$ is increasing then $Y_{t} \geq 0$ a.s. If $A:=\left\{\omega \mid Y_{t_{0}}=0\right\}$ has positive probability, then $\xi=\varphi_{t}=\int_{t}^{T} \psi_{s}^{\top} d \tilde{C}_{s}=0$ for $t_{0} \leq t \leq T$ on $A$.

Proof. As $\Gamma_{s}^{t}$ is positive a.s. the statements on nonnegativity of $Y$, both general and strict, follow from the representation as conditional expectation in (2.30). Hence it remains to prove (2.30).

$\Gamma^{t}:=\Gamma_{0}^{t}$ satisfies

$$
d \Gamma^{t}=\Gamma^{t} \gamma_{t}^{y} d t+\Gamma^{t} \alpha_{t}^{y^{\top}} d C_{t}+\Gamma^{t} \gamma_{t}^{z^{\top}} d W_{t} .
$$

Obviously $\Gamma_{s}^{t}=\Gamma^{s}\left(\Gamma^{t}\right)^{-1}$. Now apply Itô's formula to $\Gamma^{t} Y_{t}$ and write

$$
\Gamma^{T} Y_{T}-\Gamma^{t} Y_{t}=-\int_{t}^{T} \Gamma^{s} \varphi_{s} d s-\int_{t}^{T}\left(\Gamma^{s} \psi_{s}\right)^{\top} d \tilde{C}_{s}+\int_{t}^{T} \Gamma^{s}\left(Z_{s}+Y_{s} \gamma_{s}^{z}\right)^{\top} d W_{s} .
$$

Rearranging terms and taking conditional expectation gives (2.30). 
Proof of theorem 2.14. Define the bounded, progressively measurable processes

$$
\begin{aligned}
& \Delta_{x} g_{t}:=\frac{1}{Y_{t}^{1}-Y_{t}^{2}}\left(g^{1}\left(t, Y_{t}^{1}, Z_{t}^{1}\right)-g^{1}\left(t, Y_{t}^{2}, Z_{t}^{2}\right)\right) \chi_{Y_{t}^{1} \neq Y_{t}^{2}} \\
& \Delta_{x} a_{t}:=\frac{1}{Y_{t}^{1}-Y_{t}^{2}}\left(a^{1}\left(t, Y_{t}^{1}\right)-a^{1}\left(t, Y_{t}^{2}\right)\right) \chi_{Y_{t}^{1} \neq Y_{t}^{2}}
\end{aligned}
$$

and write the SDE for $\delta Y$ in the form (2.28):

$$
\begin{aligned}
d\left(\boldsymbol{\delta} Y_{t}\right)=-\left(\Delta_{x} g_{t} \boldsymbol{\delta} Y_{t}+\right. & \left.\boldsymbol{\delta} g_{t}\right) d t-\left(\boldsymbol{\delta} Y_{t} \Delta_{x} a_{t}\right)^{\top} d C_{t}^{1}-a^{1}\left(t, Y_{t}^{2}\right)^{\top} d\left(\boldsymbol{\delta} C_{t}\right) \\
& -\boldsymbol{\delta} a_{t}^{\top} d C_{t}^{2}+\left(Z_{t}^{1}-Z_{t}^{2}\right)^{\top} d W_{t}, \quad \delta Y_{T}=\xi^{1}-\xi^{2} .
\end{aligned}
$$

Here we use the notation of lemma 2.13 and set $\delta a_{t}:=a^{1}\left(t, Y_{t}^{2}\right)-a^{2}\left(t, Y_{t}^{2}\right)$. Recall that $\delta g \geq 0$ and $\tilde{C}_{t}:=\int_{0}^{t} a^{1}\left(s, Y_{s}^{2}\right)^{\top} d\left(\boldsymbol{\delta} C_{s}\right)+\int_{0}^{t} \boldsymbol{\delta} a_{s}^{\top} d C_{s}^{2}$ is increasing. Now apply lemma 2.18 to complete the proof.

\subsection{Properties of the value function}

We now turn to the value function of the control problem. Our main goal is to prove its convexity in the starting point $x$, if a convex structure as defined in (2.31) is given.

Convexity of forward-backward process Let, for $i \in\{1,2\},\left(b, \sigma, C^{i}, g, h, a\right)$ standard data for the controlled forward-backward system (2.2), and let $x^{i} \in \mathbb{R}$ and $\lambda \in[0,1]$. Denote, for $j \in\{1,2, \lambda\}$,

$$
\begin{aligned}
x^{\lambda} & =\lambda x^{1}+(1-\lambda) x^{2} & C^{\lambda} & =\lambda C^{1}+(1-\lambda) C^{2} \\
X^{j} & =X^{x^{j}, b, C^{j}, \sigma} & \left(Y^{j}, Z^{j}\right) & =(Y, Z)^{g, h\left(T, X_{T}^{j}\right), a, C^{j}}
\end{aligned}
$$

Proposition 2.19. Assume that the following holds:

$b$ is convex in the space variable $x$

$\sigma$ is linear in the space variable $x$

$h$ is convex and increasing in the space variable $x$

$g$ is convex in the variables $(x, y, z)$ and increasing in $x$

$a$ is independent of $(x, y)$

Then the following inequalities hold $\boldsymbol{P}$-a.s. for all $t \in \bar{\imath}$ :

$$
\begin{aligned}
\bar{X}_{t}^{\lambda} & :=\lambda X_{t}^{1}+(1-\lambda) X_{t}^{2} \geq X_{t}^{\lambda} \\
\bar{Y}_{t}^{\lambda} & :=\lambda Y_{t}^{1}+(1-\lambda) Y_{t}^{2} \geq Y_{t}^{\lambda}
\end{aligned}
$$

Let us first consider the forward and backward cases separately.

Forward process convexity If $b$ and $\sigma$ are linear in the space variable then obviously $\bar{X}^{\lambda}=X^{\lambda}$. The linearity assumption on $\sigma$ may not be dropped easily, as the following example shows: 
Example 2.20. Let $b=C=0$ and $\sigma(t, x)=|x|$. Then the FSDE has a unique strong solution for each initial value $x \in \mathbb{R}$. Define $X^{i}$ by

$$
X_{t}^{1}=1+\int_{0}^{t}\left|X_{s}^{1}\right| d W_{s} \quad X_{t}^{2}=-1+\int_{0}^{t}\left|X_{s}^{2}\right| d W_{s}
$$

hence

$$
X_{t}^{1}=\exp \left(W_{t}-\frac{1}{2} t\right) \quad X_{t}^{2}=-\exp \left(-W_{t}-\frac{1}{2} t\right)
$$

Let $\lambda=\frac{1}{2}$, then $X_{t}^{\lambda}=0$ is the solution of $X_{t}^{\lambda}=\int_{0}^{t}\left|X_{s}^{\lambda}\right| d W_{s}$. But

$$
2 \bar{X}_{t}^{\lambda}=\exp \left(-\frac{1}{2} t\right)\left(\exp \left(W_{t}\right)-\exp \left(-W_{t}\right)\right),
$$

so $\boldsymbol{P}\left\{\bar{X}_{t}^{\lambda}>X_{t}^{\lambda}\right\}=\boldsymbol{P}\left\{\bar{X}_{t}^{\lambda}<X_{t}^{\lambda}\right\}=\frac{1}{2}$.

Proposition 2.21. Assume that (2.31a) and (2.31b) hold. Then the solutions of the FSDE satisfy (2.32a).

Proof. We wish to apply the comparison theorem 2.8. Let

$$
\bar{b}_{t}^{\lambda}:=\lambda b\left(t, X_{t}^{1}\right)+(1-\lambda) b\left(t, X_{t}^{2}\right)
$$

the driver of $\bar{X}^{\lambda}$. Then $\bar{b}_{t}^{\lambda} \geq b\left(t, \bar{X}_{t}^{\lambda}\right)$ as $b$ is convex, which suffices according to remark.

Note. One might wonder if a generalization of proposition 2.21 to the case where $\sigma$ is convex could be acchieved by applying lemma 2.11 to the linear equation

$d\left(\bar{X}_{t}^{\lambda}-X_{t}^{\lambda}\right)=\left(\Delta_{x} b_{t}\left(\bar{X}_{t}^{\lambda}-X_{t}^{\lambda}\right)+\varepsilon_{t}\right) d t+\left\langle\Delta_{x} \sigma_{t}\left(\bar{X}_{t}^{\lambda}-X_{t}^{\lambda}\right), d W_{t}\right\rangle, \quad \bar{X}_{0}^{\lambda}-X_{0}^{\lambda}=0$.

Note however that $\Delta_{x} \sigma_{t}=\frac{1}{\bar{X}_{t}^{\lambda}-X_{t}^{\lambda}}\left(\lambda \sigma\left(t, X_{t}^{1}\right)+(1-\lambda) \sigma\left(t, X_{t}^{2}\right)-\sigma\left(t, X_{t}^{\lambda}\right)\right)$ is not necessarily bounded if $\sigma$ is not linear in $x$. This problem is not easily overcome, as the above example 2.20 shows.

Backward process convexity We first consider a more general situation than in proposition 2.19 and list some conditions ensuring that $\bar{Y}_{t}^{\lambda}-Y_{t}^{\lambda} \geq 0$ holds.

For $0 \leq \lambda \leq 1$ and $i \in\{1,2, \lambda\},\left(Y^{i}, Z^{i}\right)$ denote solutions to the BSDE with standard data $\left(g^{i}, \xi^{i}, a^{i}, C^{i}\right)$. Dependence on $x$ is not noted explicitly. We set $C^{\lambda}=\lambda C^{1}+(1-\lambda) C^{2}$ and use the notation

$$
\begin{array}{rlrl}
\bar{Y}_{t}^{\lambda} & :=\lambda Y_{t}^{1}+(1-\lambda) Y_{t}^{2} & \bar{g}_{t}^{\lambda}:=\lambda g^{1}\left(t, Y_{t}^{1}, Z_{t}^{1}\right)+(1-\lambda) g^{2}\left(t, Y_{t}^{2}, Z_{t}^{2}\right) \\
\bar{\xi}^{\lambda}:=\lambda \xi^{1}+(1-\lambda) \xi^{2} & \bar{a}_{t}^{\lambda}:=\lambda a^{1}\left(t, Y_{t}^{1}\right)+(1-\lambda) a^{2}\left(t, Y_{t}^{2}\right)
\end{array}
$$

$\bar{Y}^{\lambda}-Y^{\lambda}$ has the representation

$$
\begin{aligned}
& \bar{Y}_{t}^{\lambda}-Y_{t}^{\lambda}=E\left[\bar{\xi}^{\lambda}-\xi^{\lambda}+\int_{t}^{T} \bar{g}_{t}^{\lambda}-g^{\lambda}\left(t, Y_{t}^{\lambda}, Z_{t}^{\lambda}\right) d t\right. \\
& \left.\quad+\int_{t}^{T}\left(\lambda a^{1}\left(s, Y_{s}^{1}\right)^{\top} d C_{s}^{1}+(1-\lambda) a^{2}\left(s, Y_{s}^{2}\right)^{\top} d C_{s}^{2}-a^{\lambda}\left(s, Y_{s}^{\lambda}\right)^{\top} d C_{s}^{\lambda}\right) \mid \mathcal{F}_{t}\right]
\end{aligned}
$$

The first situation we consider is just a standard backward equation without dependence on the forward part. 
Proposition 2.22. Let $\bar{\xi}^{\lambda} \geq \xi^{\lambda}$, $a^{1}=a^{2}=a^{\lambda}$ independent of $y$ and $g^{1}=g^{2}=$ $g^{\lambda}=g$ independent of $x$. Assume that $g$ is convex in $(y, z)$. Then $\bar{Y}_{t}^{\lambda}-Y_{t}^{\lambda} \geq 0$.

This follows from the comparison theorem 2.14 with condition $(2.27 \mathrm{c})$, as $\bar{g}_{t}^{\lambda} \geq g\left(t, \bar{Y}_{t}^{\lambda}, \bar{Z}_{t}^{\lambda}\right)$ holds by convexity of $g$.

Proof of proposition 2.19. By proposition 2.21 it remains to prove (2.32b). Let

$$
\xi^{i}:=h\left(T, X_{T}^{i}\right), \quad g^{i}(t, y, z):=g\left(t, X_{t}^{i}, y, z\right) .
$$

From $(2.32 \mathrm{a})$ and $(2.31 \mathrm{c})$ we have

$$
\bar{\xi}^{\lambda}=\lambda h\left(T, X_{T}^{1}\right)+(1-\lambda) h\left(T, X_{T}^{2}\right) \geq h\left(T, \bar{X}_{T}\right) \geq h\left(T, X_{T}^{\lambda}\right)=\xi^{\lambda} .
$$

Similarly it follows from (2.32a) and (2.31d) that

$\lambda g\left(t, X_{t}^{1}, Y_{t}^{1}, Z_{t}^{1}\right)+(1-\lambda) g\left(t, X_{t}^{2}, Y_{t}^{2}, Z_{t}^{2}\right) \geq g\left(t, \bar{X}_{t}^{\lambda}, \bar{Y}_{t}^{\lambda}, \bar{Z}_{t}^{\lambda}\right) \geq g\left(t, X_{t}^{\lambda}, \bar{Y}_{t}^{\lambda}, \bar{Z}_{t}^{\lambda}\right)$.

hence $\bar{g}_{t}^{\lambda} \geq g^{\lambda}(t, y, z)$. An application of $(2.27 \mathrm{c})$ and the comparison theorem 2.14 yields $(2.32 \mathrm{~b})$.

Convexity of value $V$. We are now ready to prove the

Theorem 2.23. (Convexity of the value) Let $V_{t}\left(t_{0}, x\right)$ denote the value of the control problem 2.2 and assume that (2.31) hold. Then $V$ is convex with respect to the starting point, i.e. let $x^{1}, x^{2} \in \mathbb{R}$ and $\lambda \in[0,1]$, then

$$
V_{t}\left(t_{0}, \lambda x^{1}+(1-\lambda) x^{2}\right) \leq \lambda V_{t}\left(t_{0}, x^{1}\right)+(1-\lambda) V_{t}\left(t_{0}, x^{2}\right) .
$$

Proof. Let $\epsilon>0$ and $C^{i} \in \mathcal{A}$ such that, for $i \in\{1,2\}, Y_{t}^{t_{0}, x^{i}, C^{i}} \leq V_{t}\left(t_{0}, x^{i}\right)+\epsilon$. Obviously $C^{\lambda}:=\lambda C^{1}+(1-\lambda) C^{2}$ is in $\mathcal{A}$ and, by proposition 2.19 ,

$$
\begin{aligned}
V_{t}\left(t_{0}, \lambda x^{1}+(1-\lambda) x^{2}\right) & \leq Y_{t}^{t_{0}, x^{\lambda}, C^{\lambda}} \leq \lambda Y_{t}^{t_{0}, x^{1}, C^{1}}+(1-\lambda) Y_{t}^{t_{0}, x^{2}, C^{2}} \\
& \leq \lambda V_{t}\left(t_{0}, x^{1}\right)+(1-\lambda) V_{t}\left(t_{0}, x^{2}\right)+\epsilon .
\end{aligned}
$$

As $\epsilon$ is arbitrary the proof is complete.

We close this section with some remarks on adaptions in related problems. See also the remarks on related literature at the end of section 3.3.

Remark 2.24. The argument remains unchanged if we restrict the optimization to a convex subset $\mathcal{A}^{\prime} \subset \mathcal{A}$, as long as the admissible controls do not depend on the initial value $x$.

Remark 2.25. If $a$ depends on $x$ or $y$ it is necessary to use a more sophisticated choice of $\epsilon$-optimal controls and construction of a candidate $C^{\lambda}$. Under additional assumptions we may show e.g. that $\epsilon$-optimal states are without loss of generality monotone with respect to the starting point, i.e. if $x^{1} \leq x^{2}$ then $X^{1} \leq X^{2}$. Thus the crucial comparison theorem 2.14 can be applied to get convexity of costs for some selected controls.

Remark 2.26. In case of a generalized finite fuel condition, which can be written as $X^{a, 0,0} \leq X^{x, C^{U}, C^{L}} \leq X^{b, 0,0}$, one has to show in addition that the convex combination $C^{\lambda}$ is in fact admissible for $x^{\lambda}$. Convexity of the value function is then immediate. 


\subsection{Properties of controlled forward processes}

In this subsection we use the comparison theorem to work out a number of properties of the state process under a convex structure. We define the deflator process in the cost functional (2.5) of the Dynkin game, analyze differences of state processes and recall a result from the theory of stochastic flows on differentiability with respect to the initial condition.

The biased deflator processes $\Gamma^{x, C^{U}, C^{L}}$ for controls $\left(C^{U},-C^{L}\right)$ is defined as solution to the FSDE

$$
\begin{aligned}
d \Gamma_{t}^{x, C^{U}, C^{L}} & =\Gamma_{t}^{x, C^{U}, C^{L}}\left(D_{x}^{+} b\left(t, X_{t}^{x, C^{U}, C^{L}}\right) d t+\sigma_{x}(t)^{\top} d W_{t}\right), \\
\Gamma_{0}^{x, C^{U}, C^{L}} & =1 .
\end{aligned}
$$

Its uncontrolled version is abbreviated as $\Gamma^{x}:=\Gamma^{x, 0,0}$, which serves as deflator in the Dynkin game. We further define geometric Brownian motions $\Gamma^{u p}$ and $\Gamma^{l o}$ :

$$
\begin{array}{ll}
d \Gamma_{t}^{u p}=\Gamma_{t}^{u p} L d t+\Gamma_{t}^{u p} \sigma_{x}(t)^{\top} d W_{t}, & \Gamma_{0}^{u p}=1 \\
d \Gamma_{t}^{l o}=-\Gamma_{t}^{l o} L d t+\Gamma_{t}^{l o} \sigma_{x}(t)^{\top} d W_{t}, & \Gamma_{0}^{l o}=1 .
\end{array}
$$

Here $L$ is a Lipschitz constant for $b$ These processes form universal bounds for "difference quotients" and differentials of the forward process.

Lemma 2.27. Let $(b, \sigma, C)$ standard data of the FSDE satisfying (2.31a) and (2.31b). Then the following estimates hold for all $t \in \bar{\imath}$ almost surely:

$$
\begin{aligned}
& X_{t}^{x+\delta, C^{U}, 0}-X_{t}^{x, C^{U}, 0} \leq X_{t}^{x+\delta, 0,0}-X_{t}^{x, 0,0} \leq X_{t}^{x+\delta, 0, C^{L}}-X_{t}^{x, 0, C^{L}} \\
& \delta \Gamma_{t}^{l o} \leq \delta \Gamma_{t}^{x, C^{U}, C^{L}} \leq X_{t}^{x+\delta, C^{U}, C^{L}}-X_{t}^{x, C^{U}, C^{L}} \leq \delta \Gamma_{t}^{x+\delta, C^{U}, C^{L}} \leq \delta \Gamma_{t}^{u p}
\end{aligned}
$$

and $X_{t}^{x+\delta, C^{U}, 0}-X_{t}^{x, C^{U}, 0} \geq 0$ and $\Gamma_{t}^{l o}>0$. Furthermore we have that

$$
\lim _{\delta \searrow 0} \frac{1}{\delta}\left(X_{t}^{x+\delta, C^{U}, C^{L}}-X_{t}^{x, C^{U}, C^{L}}\right)=\Gamma_{t}^{x, C^{U}, C^{L}}
$$

Proof. Nonnegativity of the first term in (2.37) follows from the comparison theorem 2.8, as both processes have the same driver and controls.

For the first inequality in (2.37), define the stopping time

$$
\rho:=\inf \left\{t \in \bar{\imath} \mid X_{t}^{x+\delta, C^{U}, 0} \leq X_{t}^{x, 0,0}\right\}
$$

and observe that, due to the left-continuity of both processes and the comparison theorem 2.8, $\rho$ separates the intervalls where the difference of these processes is positive and negative respectively:

$$
\begin{array}{ll}
X_{t}^{x+\delta, C^{U}, 0} \geq X_{t}^{x, 0,0} & \text { if } t \leq \rho \\
X_{t}^{x+\delta, C^{U}, 0} \leq X_{t}^{x, 0,0} & \text { if } t>\rho .
\end{array}
$$

Now set $\hat{X}^{1}:=X^{x+\delta, 0,0}-X^{x, 0,0}$ and $\hat{X}^{2}:=X^{x+\delta, C^{U}, 0}-X^{x, C^{U}, 0}$. For $t \leq \rho$ consider $\tilde{X}^{1}:=X^{x+\delta, 0,0}-X^{x+\delta, C^{U}, 0}$ and $\tilde{X}^{2}:=X^{x, 0,0}-X^{x, C^{U}, 0}$. As $\hat{X}^{1}-\hat{X}^{2}=$ $\tilde{X}^{1}-\tilde{X}^{2}$ it suffices to prove $\tilde{X}^{1} \geq \tilde{X}^{2}$. Both processes are nonnegative, have 
the same starting point 0 and control process 0 . Their volatility processes are $\sigma\left(s, \tilde{X}_{s}^{1}\right)$ and $\sigma\left(s, \tilde{X}_{s}^{2}\right)$. Their drivers $\tilde{b}^{1}$ and $\tilde{b}^{2}$ satisfy

$$
\begin{array}{ll}
\tilde{b}_{s}^{1}=b\left(s, X_{s}^{x+\delta, 0,0}\right)-b\left(s, X_{s}^{x+\delta, C^{U}, 0}\right) & \geq D_{x}^{+} b\left(s, X_{s}^{x+\delta, C^{U}, 0}\right) \tilde{X}_{s}^{1} \\
\tilde{b}_{s}^{2}=b\left(s, X_{s}^{x, 0,0}\right)-b\left(s, X_{s}^{x, C^{U}, 0}\right) & \leq D_{x}^{-} b\left(s, X_{s}^{x, 0,0}\right) \tilde{X}_{s}^{2}
\end{array}
$$

due to the convexity of $b$ in the space variable. As $D_{x}^{+} b\left(s, X_{s}^{x+\delta, C^{U}, 0}\right) \geq$ $D_{x}^{-} b\left(s, X_{s}^{x, 0,0}\right)$ for $s \leq \rho$, the first inequality in (2.37) for $t \leq \rho$ follows from the comparison theorem 2.8 and remark 2.9 .

For $t>\rho$ consider $\hat{X}^{1}$ and $\hat{X}^{2}$. They are nonnegative, satisfy $\hat{X}_{\rho}^{1}-\hat{X}_{\rho}^{2} \geq 0$ by above argument and have the same control process 0 . Their volatility processes are $\sigma\left(s, \hat{X}_{s}^{1}\right)$ and $\sigma\left(s, \hat{X}_{s}^{2}\right)$. Their drivers $\hat{b}^{1}$ and $\hat{b}^{2}$ satisfy

$$
\begin{aligned}
& \hat{b}_{s}^{1}=b\left(s, X_{s}^{x+\delta, 0,0}\right)-b\left(s, X_{s}^{x, 0,0}\right) \geq D_{x}^{+} b\left(s, X_{s}^{x, 0,0}\right) \hat{X}_{s}^{1} \\
& \hat{b}_{s}^{2}=b\left(s, X_{s}^{x+\delta, C^{U}, 0}\right)-b\left(s, X_{s}^{x, C^{U}, 0}\right) \leq D_{x}^{-} b\left(s, X_{s}^{x+\delta, C^{U}, 0}\right) \hat{X}_{s}^{2},
\end{aligned}
$$

due to the convexity of $b$ in the space variable. Then $\hat{X}_{t}^{1} \geq \hat{X}_{t}^{2}$ for $t>\rho$ follows from $D_{x}^{+} b\left(s, X_{s}^{x, 0,0}\right) \geq D_{x}^{-} b\left(s, X_{s}^{x+\delta, C^{U}, 0}\right)$ and remark 2.9. This completes the proof of the first inequality in (2.37).

For the second inequality consider the stopping time

$$
\varrho:=\inf \left\{t \in \bar{\imath} \mid X_{t}^{x+\delta, 0,0} \leq X_{t}^{x, 0, C^{L}}\right\}
$$

and define the processes $\bar{X}^{1}:=X^{x+\delta, 0, C^{L}}-X^{x+\delta, 0,0}, \bar{X}^{2}:=X^{x, 0, C^{L}}-X^{x, 0,0}$, $\check{X}^{1}:=X^{x+\delta, 0, C^{L}}-X^{x, 0, C^{L}}$ and $\check{X}^{2}:=X^{x+\delta, 0,0}-X^{x, 0,0}$. Analogous arguments as employed for the first inequality show that $\bar{X}^{1}-\dot{X}^{2} \geq 0$ for $t \leq \varrho$ and $\check{X}^{1}-\check{X}^{2} \geq 0$ for $t>\varrho$, which are equivalent to the second inequality in (2.37).

The proof of (2.38) rests on quite similar arguments. $\Gamma^{l o}>0 \boldsymbol{P}$-a.s. is a standard property of geometric Brownian motion. The first and last inequality follow from the Lipschitz property of $b$, linearity of $\sigma$ and the comparison theorem 2.8. For the second and third inequality use convexity of $b$ in $x$, which gives the estimate

$$
\begin{aligned}
& D_{x}^{+} b\left(t, X_{t}^{x, C^{U}, C^{L}}\right)\left(X_{t}^{x+\delta, C^{U}, C^{L}}-X_{t}^{x, C^{U}, C^{L}}\right) \\
& \leq b\left(t, X_{t}^{x+\delta, C^{U}, C^{L}}\right)-b\left(t, X_{t}^{x, C^{U}, C^{L}}\right) \\
& \leq D_{x}^{+} b\left(t, X_{t}^{x+\delta, C^{U}, C^{L}}\right)\left(X_{t}^{x+\delta, C^{U}, C^{L}}-X_{t}^{x, C^{U}, C^{L}}\right),
\end{aligned}
$$

and apply, again, the comparison theorem.

(2.39) is a standard property of stochastic flows, see e.g. (cf. [51], [31]. We give a short outline of the argument in our situation. In view of (2.38) it suffices to prove

$$
\Gamma_{t}^{x+\delta, C^{U}, C^{L}} \searrow \quad \Gamma_{t}^{x, C^{U}, C^{L}} \quad \text { as } \delta \searrow 0 .
$$

It is obvious from the comparison theorem 2.8 that $X_{t}^{x+\delta, C^{U}, C^{L}}$ and hence $\Gamma_{t}^{x+\delta, C^{U}, C^{L}}$ decrease as $\delta \searrow 0$. By the dominated convergence theorem, with

$\left\|\boldsymbol{\delta} D_{x}^{+} b\right\|_{2}^{2}:=E\left[\int_{0}^{T}\left(D_{x}^{+} b\left(s, X_{s}^{x+\delta, C^{U}, C^{L}}\right)-D_{x}^{+} b\left(s, X_{s}^{x, C^{U}, C^{L}}\right)\right)^{2}\left|\Gamma_{s}^{x, C^{U}, C^{L}}\right|^{2} d s\right]$, 
$\left\|\boldsymbol{\delta} D_{x}^{+} b\right\|_{2}^{2} \stackrel{\delta \searrow 0}{\longrightarrow} 0$ Hence $E\left[\sup _{0 \leq s \leq T}\left|\Gamma_{s}^{x+\delta, C^{U}, C^{L}}-\Gamma_{s}^{x, C^{U}, C^{L}}\right|^{2}\right]$ converges to zero as the a priori estimates of proposition 2.6 show. Hence $\Gamma_{t}^{x+\delta, C^{U}, C^{L}}-\Gamma_{t}^{x, C^{U}, C^{L}}$ also converges uniformly in $t \boldsymbol{P}$-a.s. to zero.

Remark 2.28. If $b$ is convex, $b_{x}$ exists a.e. Hence as long as $X^{x, C^{U}, C^{L}}$ is not deterministic we can replace $D_{x}^{+} b$ by $b_{x}$.

\section{Historical remarks}

Our presentation of a priori estimates and comparison theorems is along the lines of EL Karoui and Quenez [24], and PENG [49], where similar results are used to prove existence and uniqueness of solutions to BSDE. The method of translation to a control-free SDE employed in our proof of existence and uniqueness is borrowed from PENG [49]. The argument in theorem 2.23 is taken from Karatzas and Shreve [35]. 


\section{Deriving the stopping problem}

In addition to conditions (2.31), we now require the data of the FBSDE (2.2) to satisfy also:

$$
\begin{aligned}
& b \text { is differentiable in } x \\
& h \text { is differentiable in } x
\end{aligned}
$$

$g$ is partially differentiable in $x, y$ and $z$

$g_{y}$ is increasing in $x$

$g_{z}$ is independent of $x, y, z$

Both components of $a$ are nonnegative and continuous.

If (3.1e) is assumed we drop dependence on $z$ in the notation for partial derivatives of $g$.

Our main result of this section is the following

Theorem 3.1. Assume that (2.31) and (3.1) hold and that there exists an optimal control $C=\left(C^{U},-C^{L}\right)$ for the control problem 2.2 at the starting point $\left(t_{0}, x\right)$. Then the value function of the control problem $V$ is partially differentiable at $x$ with respect to the space variable. The associated Dynkin game 2.5 has a solution, and its value is the partial derivative of the value of the control problem:

$$
\frac{\partial}{\partial x} V_{t}\left(t_{0}, x\right)=u_{t}\left(t_{0}, x\right) \quad \text { for } t_{0} \leq t \leq \sigma^{0} \wedge \tau^{0} \boldsymbol{P} \text {-a.s.. }
$$

Here $\sigma^{0}:=\inf \left\{t \geq t_{0} \mid C_{t}^{U}>C_{t_{0}}^{U}\right\}$ and $\tau^{0}:=\inf \left\{t \geq t_{0} \mid C_{t}^{L}>C_{t_{0}}^{L}\right\}$ are the first action times of the controls $C^{U}$ and $C^{L}$. They form a saddle point for the Dynkin game which has value

$$
u_{t}\left(t_{0}, x\right)=R_{t}^{t_{0}, x}\left(\sigma^{0}, \tau^{0}\right) \quad \text { for } t_{0} \leq t \leq \sigma^{0} \wedge \tau^{0} \boldsymbol{P} \text {-a.s. }
$$

The proof essentially rests on the four relations

$$
\begin{aligned}
u_{t}^{-}\left(t_{0}, x\right) & \leq u_{t}^{+}\left(t_{0}, x\right), & \Delta_{-} V_{t}\left(t_{0}, x\right) & \leq \Delta^{+} V_{t}\left(t_{0}, x\right), \\
\Delta^{+} V_{t}\left(t_{0}, x\right) & \leq u_{t}^{-}\left(t_{0}, x\right), & u_{t}^{+}\left(t_{0}, x\right) & \leq \Delta_{-} V_{t}\left(t_{0}, x\right) .
\end{aligned}
$$

Here $\Delta^{+} V$ and $\Delta_{-} V$ denote the upper right and lower left Dini derivatives of the value function $V$. The first inequality follows from the definitions (2.6), the second from theorem 2.23. The third and fourth are the subject of sections 3.1 and 3.2.

For our investigation of the value function of the control problem we choose an optimal trajectory and a state processes that tracks it from a nearby starting point, up to a pair of stopping times. In a pathwise analysis it is possible to construct controls such that the difference of costs resembles the payoff of a stochastic game of optimal stopping. Thus we establish correspondence between "difference quotients" of the control cost process and the cost of stopping times.

The formulation of tracking and the analysis of cost behaviour is carried out without reference to specific properties of the trajectory tracked, but to draw conclusions on the value process we will have to assume that an optimal policy exists. We start in section 3.1 with a discussion of right hand side difference 
quotients. The left hand side difference quotient is almost parallel and there is a brief outline in section 3.2 of the steps necessary. The section closes with proof of the main theorem and a discussion of possible extensions and related results.

\subsection{Upper right Dini derivative}

First we will introduce the construction of a tracking process. We recall some properties of the state processes in this situation. Then we analyze characteristics of the control involved and of the cost functionals, restricting our assumptions to (3.10) below.

Making use of the convexity of the data and its consequences presented in lemma 2.27 we then find estimates for the difference quotient of cost functionals and investigate its limit behaviour. This enables us to find an estimate for the upper right Dini derivative of the value function $V$ in terms of the value function of a Dynkin game.

Construction of the tracking process from above We consider a controlled process $X^{\star}=X^{t_{0}, x, C^{U}, C^{L}}$ and a process $X^{\delta}$ tracking $X^{\star}$ from above.

Define the crossing time

$$
\tau^{\delta}:=\inf \left\{t \geq t_{0} \mid X_{t}^{t_{0}, x+\delta, C^{U}, 0} \leq X_{t}^{t_{0}, x, C^{U}, C^{L}}\right\} .
$$

and let $\sigma \in \mathcal{T}_{t_{0}}$. The tracking process $X^{\delta}$ for the starting point $\left(t_{0}, x+\delta\right)$ is defined by

$$
X_{t}^{\delta}:= \begin{cases}X_{t}^{t_{0}, x+\delta, C^{U}, 0} & t \leq \sigma \wedge \tau^{\delta} \\ X_{t}^{t_{0}, x, C^{U}, C^{L}} & t>\sigma \wedge \tau^{\delta}\end{cases}
$$

which has parallel upper control as the state traced and no lower control until $\sigma$ occurs or $X^{t_{0}, x, C^{U}, C^{L}}$ crosses its trajectory.

Let us suppress dependence on $t_{0}$ in the notation. We want to obtain $X^{\delta}$ as controlled process $X^{x+\delta, C^{\delta, U}, C^{\delta, L}}$ to compare the costs associated with $X^{\delta}$ and $X^{\star}$. To acchieve this, define

$$
\begin{aligned}
C_{t}^{\delta, U} & := \begin{cases}C_{t}^{U} & t \leq \sigma \\
C_{t}^{U}+X_{\sigma}^{x+\delta, C^{U}, 0}-X_{\sigma}^{x, C^{U}, C^{L}} & t>\sigma, \sigma \leq \tau^{\delta} \\
C_{t}^{U} & t>\sigma, \sigma>\tau^{\delta}\end{cases} \\
C_{t}^{\delta, L} & := \begin{cases}0 & t \leq \sigma \wedge \tau^{\delta} \\
C_{t}^{L}-C_{\sigma}^{\lambda} & t>\sigma, \sigma \leq \tau^{\delta} \\
C_{t}^{L}-C_{\tau^{\delta}}^{L}-\left(X_{\tau^{\delta}}^{x+\delta, C^{U}, 0}-X_{\tau^{\delta}}^{x, C^{U}, C^{L}}\right) & t>\tau^{\delta}, \sigma>\tau^{\delta}\end{cases}
\end{aligned}
$$

Remark 3.2. We have to check that $C^{\delta, L}$ is increasing at $\tau^{\delta}$ : Consider $t>\tau^{\delta}$ 
on $\left\{\sigma>\tau^{\delta}\right\}$. Then by definition of $\tau^{\delta}$

$$
\begin{aligned}
& 0 \leq C_{t}^{L}-C_{\tau^{\delta}+}^{L}+X_{\tau^{\delta}+}^{x, C^{U}, C^{L}}-X_{\tau^{\delta}+}^{x+\delta, C^{U}, 0} \\
& =C_{t}^{L}-C_{\tau^{\delta}+}^{L}+X_{\tau^{\delta}}^{x, C^{U}, C^{L}}-\left(C_{\tau^{\delta}+}^{U}-C_{\tau^{\delta}}^{U}\right) \\
& \quad+\left(C_{\tau^{\delta}+}^{L}-C_{\tau^{\delta}}^{L}\right)-\left(X_{\tau^{\delta}}^{x+\delta, C^{U}, 0}-\left(C_{\tau^{\delta}+}^{U}-C_{\tau^{\delta}}^{U}\right)\right) \\
& \quad=C_{t}^{L}-C_{\tau^{\delta}}^{L}-\left(X_{\tau^{\delta}}^{x+\delta, C^{U}, 0}-X_{\tau^{\delta}}^{x, C^{U}, C^{L}}\right)
\end{aligned}
$$

Hence $C^{\delta}:=\left(C^{\delta, U},-C^{\delta, L}\right)$ is in $\mathcal{A}$.

Proposition 3.3. Let $C^{\delta} \in \mathcal{A}$ as defined in (3.6). Then $X^{x+\delta, C^{\delta, U}, C^{\delta, L}}=X^{\delta}$ and

$$
0 \leq X_{t}^{x+\delta, C^{\delta, U}, C^{\delta, L}}-X_{t}^{x, C^{U}, C^{L}} \leq X_{t}^{x+\delta, C^{U}, 0}-X_{t}^{x, C^{U}, 0} .
$$

Further $Y_{t}^{x+\delta, C^{\delta, U}, C^{\delta, L}}=Y_{t}^{x, C^{U}, C^{L}}$ and $Z_{t}^{x+\delta, C^{\delta, U}, C^{\delta, L}}=Z_{t}^{x, C^{U}, C^{L}}$ for $\sigma \wedge \tau^{\delta}<$ $t \leq T$.

Proof. $X_{t}^{\delta}=X_{t}^{x+\delta, C^{\delta, U}, C^{\delta, L}}$ for $t \leq \sigma \wedge \tau^{\delta}$ is obvious from the construction. Consider the right hand side limit $X_{\sigma \wedge \tau^{\delta}+}^{x+\delta, C^{\delta, U}, C^{\delta, L}}$ which equals $X_{\sigma \wedge \tau^{\delta}+}^{x, C^{U}, C^{L}}$ by definition of $C^{\delta}$, hence $X_{t}^{x+\delta, C^{\delta, U}, C^{\delta, L}}=X_{t}^{\delta}$ for $t>\sigma \wedge \tau^{\delta}$. The equalities for $Y^{x, C^{U}, C^{L}}$ and $Z^{x, C^{U}, C^{L}}$ on $\left(\sigma \wedge \tau^{\delta}, T\right]$ follow from the uniqueness of solutions to BSDE.

It remains to prove (3.7). The first inequality follows from (3.5) and the definition of $\tau^{\delta}$. Observe that $X^{x, C^{U}, C^{L}} \geq X^{x, C^{U}, 0}$ from the comparison theorem 2.8. For $t \leq \sigma \wedge \tau^{\delta}$ the second inequality then follows from the definition of $C^{\delta}$. Further $X_{t}^{x+\delta, C^{\delta, U}, C^{\delta, L}}=X_{t}^{x, C^{U}, C^{L}}$ for $t>\sigma \wedge \tau^{\delta}$; by lemma 2.27 the right hand side is nonnegative.

We use, $\forall t \in \bar{\imath}$, the notation

$$
\begin{array}{rlrl}
X_{t}^{\star}:=X_{t}^{x, C^{U}, C^{L},}, & Y_{t}^{\star}:=Y_{t}^{x, C^{U}, C^{L},} & Z_{t}^{\star}:=Z_{t}^{x, C^{U}, C^{L},} \\
Y_{t}^{\delta}:=Y_{t}^{x+\delta, C^{\delta, U}, C^{\delta, L},}, & Z_{t}^{\delta}:=Z_{t}^{x+\delta, C^{\delta, U}, C^{\delta, L}}
\end{array}
$$

$\frac{1}{\delta}\left(Y^{\delta}-Y^{\star}\right)$ will be the difference quotient under observation later in this section.

Some characteristics of the tracking process Next define the first action time $\tau^{0}$ of $C^{L}$ by

$$
\tau^{0}:=\inf \left\{t \geq t_{0} \mid X_{t}^{x, C^{U}, 0}<X_{t}^{\star}\right\}=\inf \left\{t \geq t_{0} \mid C_{t}^{L}>C_{t_{0}}^{L}\right\} .
$$

Remark 3.4. Observe that $\tau^{\delta} \searrow \tau^{0}$ almost surely. This follows from (3.11) below and existence of right hand limits for all processes involved.

Further, if $C_{\tau^{0}+}^{L}(\omega)>C_{\tau^{0}}^{L}(\omega)$ there is a $\delta_{0}(\omega)$ such that $\tau^{\delta}(\omega)=\tau^{0}(\omega)$ for all $\delta \leq \delta_{0}$ almost surely. This again follows from (3.11) if we set $\delta_{0}:=$ $\left(C_{\tau^{0}+}^{L}-C_{\tau^{0}}^{L}\right) / \Gamma_{\tau^{0}}^{u p}$. 
Remark 3.5. Assume (2.31a) and (2.31b) hold. Then for $t \in \bar{\imath}$

$$
\delta \Gamma_{t}^{x, C^{U},{ }^{0}} \chi_{t \leq \sigma \wedge \tau^{0}} \leq X_{t}^{\delta}-X_{t}^{\star} \leq \delta \Gamma_{t}^{x+\delta, C^{U}, 0} \chi_{t \leq \sigma \wedge \tau^{\delta}} \quad \boldsymbol{P} \text {-a.s. }
$$

This follows from the convexity of $b$ and definition of $\tau^{0}, \tau^{\delta}$ and $X^{\delta}$ as in lemma 2.27 .

At the end of this section we will assume that $\left(C^{U}, C^{L}\right)$ is in fact optimal for the control problem, but this is of no importance in the development of the next results.

In the discussion of $C^{L}$ and $Y^{\delta}-Y^{\star}$ we can reduce our assumptions in (2.31) and (3.1) to

$b$ is differentiable in $x$

$\sigma$ is linear in the space variable $x$

$h$ is increasing in the space variable $x$

$g$ is increasing in the space variable $x$

Both components of $a$ are nonnegative

Restricting to these assumptions will facilitate a transfer to the situation where the left hand side derivative of $V$ is considered.

We reformulate some results on controlled processes of lemma 2.27 for our situation.

Lemma 3.6. Assume (3.10b) holds. Then for any $C \in \mathcal{A}$ and initial values $x, x+\delta$ the estimate

$$
0<\delta \Gamma_{t}^{l o} \leq X_{t}^{x+\delta, C^{U}, C^{L}}-X_{t}^{x, C^{U}, C^{L}} \leq \delta \Gamma_{t}^{u p}
$$

holds, $X_{t}^{x+\delta, C^{U}, C^{L}}$ decreases as $\delta$ decreases and proposition 3.3 remains valid. Furthermore, $X^{\delta}$ decreases to $X^{\star}$.

These are consequences of the Lipschitz property of the data $b$ and $\sigma$, linearity of $\sigma$ and the comparison theorem 2.8. The proof of proposition 3.3 does not require convexity of the data.

Bounds for $C^{L} \quad$ We wish to give an estimate for $C_{t}^{L}$ if $t \leq \tau^{\delta}$. We derive this from the definition of $\tau^{\delta}$ in the following way: Let $\tilde{\Theta}_{t}(\omega) \in\left[X_{t}^{x, C^{U}, 0}(\omega), X_{t}^{\star}(\omega)\right] \subset$ $\mathbb{R}$ such that

$$
b_{x}\left(t, \tilde{\Theta}_{t}\right)\left(X_{t}^{\star}-X_{t}^{x, C^{U}, 0}\right)=b\left(t, X_{t}^{\star}\right)-b\left(t, X_{t}^{x, C^{U}, 0}\right) \quad \boldsymbol{P} \text {-a.s. }
$$

and let $\Gamma_{t}^{\tilde{\Theta}}$ the solution of the linear SDE

$$
d \Gamma_{t}^{\tilde{\Theta}}=b_{x}\left(t, \tilde{\Theta}_{t}\right) \Gamma_{t}^{\tilde{\Theta}} d t+\sigma\left(t, \Gamma_{t}^{\tilde{\Theta}}\right)^{\top} d W_{t}, \quad \Gamma_{0}^{\tilde{\Theta}}=1 .
$$

It is obvious from the comparison theorem 2.8 and Lipschitz continuity of $b$ that $\Gamma_{t}^{l o} \leq \Gamma_{t}^{\tilde{\Theta}} \leq \Gamma_{t}^{u p}$ a.s. Hence $\Gamma_{t}^{\tilde{\Theta}} \in \mathbb{R}_{>0}$ for $t \leq \tau^{\delta}$ a.s. Now consider 
$X^{\star}-X^{x, C^{U}, 0}$ : Apply the Itô formula to $\left(\Gamma_{t}^{\tilde{\Theta}}\right)^{-1}\left(X_{t}^{\star}-X_{t}^{x, C^{U}, 0}\right)$ and recall that this equals zero for $t \leq \tau^{0}$ to get the representation - similar to lemma $2.11-$

$$
\begin{aligned}
X_{t}^{\star}-X_{t}^{x, C^{U}, 0}= & \int_{0}^{t} b_{x}\left(s, \tilde{\Theta}_{s}\right)\left(X_{s}^{\star}-X_{s}^{x, C^{U}, 0}\right) d s+C_{t}^{L} \\
& +\int_{0}^{t} \sigma\left(s, X_{s}^{\star}-X_{s}^{x, C^{U}, 0}\right)^{\top} d W_{s} \quad=\int_{\tau^{0}}^{\tau^{0} \vee t} \Gamma_{t}^{\tilde{\Theta}}\left(\Gamma_{s}^{\tilde{\Theta}}\right)^{-1} d C_{s}^{L}
\end{aligned}
$$

This leads to the following estimate for $\tau^{0}<t \leq \tau^{\delta}$ :

$$
\begin{aligned}
\left(\sup _{\tau^{0}<s \leq t} \Gamma_{s}^{\tilde{\Theta}}\right)^{-1} \Gamma_{t}^{\tilde{\Theta}} C_{t}^{L} & =\left(\inf _{\tau^{0}<s \leq t}\left(\Gamma_{s}^{\tilde{\Theta}}\right)^{-1}\right) \Gamma_{t}^{\tilde{\Theta}} C_{t}^{L} \\
& \leq \int_{\tau^{0}}^{t} \Gamma_{t}^{\tilde{\Theta}}\left(\Gamma_{s}^{\tilde{\Theta}}\right)^{-1} d C_{s}^{L}=X_{t}^{\star}-X_{t}^{x, C^{U}, 0} \\
& \leq X_{t}^{x+\delta, C^{U}, 0}-X_{t}^{x, C^{U}, 0} \leq \delta \Gamma_{t}^{u p}
\end{aligned}
$$

Similarly we obtain a lower bound on $C_{t}^{L}$ for $t$ with $C_{t}^{L}>0$, i.e. $t>\tau^{0}$. The results are summarized in the following

Lemma 3.7. Let $\tau^{0}$ and $\tau^{\delta}$ as defined in (3.8) and (3.4), and assume (3.10) holds. Then $C_{t}^{L}$ has $\boldsymbol{P}$-a.s. upper and lower bounds for $\tau^{0}<t \leq \tau^{\delta}$ :

$$
\left(X_{t}^{\star}-X_{t}^{x, C^{U}, 0}\right) \frac{1}{\Gamma_{t}^{\tilde{\Theta}}}\left(\inf _{\tau^{0}<s \leq t} \Gamma_{s}^{\tilde{\Theta}}\right) \leq C_{t}^{L} \leq\left(X_{t}^{\star}-X_{t}^{x, C^{U}, 0}\right) \frac{1}{\Gamma_{t}^{\tilde{\Theta}}}\left(\sup _{\tau^{0}<s \leq t} \Gamma_{s}^{\tilde{\Theta}}\right) .
$$

Thus $C^{L}$ satisfies

$$
C_{t}^{L} \leq \delta \Gamma_{t}^{u p}\left(\Gamma_{t}^{l o}\right)^{-1}\left(\sup _{\tau^{0} \leq s \leq t} \Gamma_{s}^{u p}\right)=: \delta \cdot \overline{C_{t}^{L, \delta}} \quad \text { for } t \leq \tau^{\delta} \boldsymbol{P} \text {-a.s. }
$$

Remark 3.8. Assume that (2.31) and (3.1) hold. Then

$$
\Gamma_{t}^{x, C^{U}, 0} \leq \Gamma_{t}^{\tilde{\Theta}} \leq \Gamma_{t}^{x+\delta, C^{U}, 0}
$$

for $t \leq \tau^{\delta}$ by convexity of $b$ and the comparison theorem 2.8 .

Limit behaviour of cost functionals Our plan is to find bounds for the process $\frac{1}{\delta}\left(Y^{\delta}-Y^{\star}\right)$, which will result in a first connection with a Dynkin game. But before we can investigate this "difference quotient" we need more information about the processes $Y^{\delta}$ and their behaviour as $\delta \searrow 0$. We start with

Proposition 3.9. Assume that (3.10) holds. Let, for $\delta, \delta^{\prime} \in \mathbb{R}_{>0}$, the processes $Y^{\delta}, Y^{\delta^{\prime}}$ as defined in proposition 3.3. Then

$$
Y_{t}^{\star} \leq Y_{t}^{\delta} \leq Y_{t}^{\delta^{\prime}} \quad \text { for } \delta \leq \delta^{\prime} \text { for all } t \boldsymbol{P} \text {-a.s. }
$$

Proof. Let us look at the inequality $Y_{t}^{\star} \leq Y_{t}^{\delta}$ first. For $t>\sigma \wedge \tau^{\delta}$ equality holds by definition of $\left(C^{\delta, U}, C^{\delta, L}\right)$. 
Now let $t \leq \sigma \wedge \tau^{\delta}$. We wish to apply the comparison theorem 2.14. From proposition 3.3 and the properties (3.10c) and (3.10d) of $h, g$ it follows that

$$
h\left(X_{T}^{\delta}\right) \geq h\left(X_{T}^{\star}\right), \quad g\left(t, X_{t}^{\delta}, y, z\right) \geq g\left(t, X_{t}^{\star}, y, z\right) .
$$

Consider the difference of control processes $\delta C:=\left(C^{\delta, U}-C^{U},-\left(C^{\delta, L}-C^{L}\right)\right)$. The components have the representation

$$
\begin{aligned}
& C_{t}^{\delta, U}-C_{t}^{U}=\left(X_{\sigma}^{\delta}-X_{\sigma}^{\star}\right) \boldsymbol{\chi}_{\substack{t>\sigma \\
\sigma \leq \tau^{\delta}}}, \\
& C_{t}^{\delta, L}-C_{t}^{L}=-C_{t}^{L} \boldsymbol{\chi}_{t \leq \sigma \wedge \tau^{\delta}}-C_{\sigma}^{L} \boldsymbol{\chi}_{\substack{t>\sigma \wedge \tau^{\delta} \\
\sigma \leq \tau^{\delta}}}-\left(C_{\tau^{\delta}}^{L}+\left(X_{\tau^{\delta}}^{\delta}-X_{\tau^{\delta}}^{\star}\right)\right) \boldsymbol{\chi}_{\substack{t>\sigma \wedge \tau^{\delta} \\
\sigma>\tau^{\delta}}} .
\end{aligned}
$$

Recall the argument in remark 3.2 to see that both components of $\delta C$ are increasing, and therefore $\int_{[0, t)} a_{s}^{\top} d\left(\delta C_{s}\right)$ is increasing in $t$.

So the assumptions of the comparison theorem 2.14 are satisfied, which completes the proof of the first inequality.

For the second inequality observe that we are done if $t>\sigma \wedge \tau^{\delta}$, as then $Y_{t}^{\delta}=Y_{t}^{\star}$. For $t \leq \sigma \wedge \tau^{\delta}$ the processes satisfy

$$
\begin{aligned}
Y_{t}^{\delta^{\prime}}-Y_{t}^{\delta}= & Y_{\sigma \wedge \tau^{\delta}}^{\delta^{\prime}}-Y_{\sigma \wedge \tau^{\delta}}^{\delta}+\int_{t}^{\sigma \wedge \tau^{\delta}} g\left(s, X_{s}^{\delta^{\prime}}, Y_{s}^{\delta^{\prime}}, Z_{s}^{\delta^{\prime}}\right)-g\left(s, X_{s}^{\delta}, Y_{s}^{\delta}, Z_{s}^{\delta}\right) d s \\
& -\int_{t}^{\sigma \wedge \tau^{\delta}} Z_{s}^{\delta^{\prime}}-Z_{s}^{\delta} d W_{s} .
\end{aligned}
$$

Hence it suffices to prove $Y_{\sigma \wedge \tau^{\delta}}^{\delta^{\prime}} \geq Y_{\sigma \wedge \tau^{\delta}}^{\delta}$, as $g\left(s, X_{s}^{\delta^{\prime}}, y, z\right) \geq g\left(s, X_{s}^{\delta}, y, z\right)$ and an application of the comparison theorem 2.14 will complete the argument.

To this end use a slightly different representation (cf. (3.13) below):

$$
\begin{aligned}
& Y_{\sigma \wedge \tau^{\delta}}^{\delta}-Y_{\sigma \wedge \tau^{\delta}}^{\star}=\left(h\left(X_{T}^{\delta}\right)-h\left(X_{T}^{\star}\right)\right) \chi_{\sigma \wedge \tau^{\delta}=T}+a_{\sigma}^{U}\left(X_{\sigma}^{\delta}-X_{\sigma}^{\star}\right) \chi_{\substack{\sigma \leq \tau^{\delta} \\
\sigma<T}} \\
& +a_{\tau^{\delta}}^{L}\left(X_{\tau^{\delta}}^{\delta}-X_{\tau^{\delta}}^{\star}\right) \chi_{\tau^{\delta}<\sigma} \\
& \leq\left(h\left(X_{T}^{\delta^{\prime}}\right)-h\left(X_{T}^{\star}\right)\right) \chi_{\sigma \wedge \tau^{\delta}=T}+a_{\sigma}^{U}\left(X_{\sigma}^{\delta^{\prime}}-X_{\sigma}^{\star}\right) \chi_{\substack{\sigma \leq \tau^{\delta} \\
\sigma<T}} \\
& +a_{\tau^{\delta}}^{L}\left(C_{\tau^{\delta}+}^{L}-C_{\tau^{\delta}}^{L}\right) \chi_{\substack{\tau^{\delta}<\sigma \\
\tau^{\delta}<T}}+\left(Y_{\sigma \wedge \tau^{\delta}+}^{\delta^{\prime}}-Y_{\sigma \wedge \tau^{\delta}+}^{\star}\right) \chi_{\tau_{\tau^{\delta}<\sigma}<T} \\
& =Y_{\sigma \wedge \tau^{\delta}}^{\delta^{\prime}}-Y_{\sigma \wedge \tau^{\delta}}^{\star}
\end{aligned}
$$

where the inequality is due to the definition of $C^{\delta, L}$ and remark $3.2, Y^{\delta^{\prime}} \geq Y^{\star}$ and (3.10c), (3.10e) and the monotonicity of $X^{\delta}$ in $\delta$. The last equality follows from $\tau^{\delta} \leq \tau^{\delta^{\prime}}$. This completes the proof.

Observe that we can interprete $Y^{\delta}-Y^{\star}$ as solution to a BSDE where the terminal value is $\mathcal{F}_{\sigma \wedge \tau^{\delta}}$-measurable. In this form it resembles the payoff of a stochastic game of optimal stopping:

$$
\begin{aligned}
Y_{t}^{\delta}-Y_{t}^{\star}=E & {\left[\int_{t}^{\sigma \wedge \tau^{\delta}} g\left(s, X_{s}^{\delta}, Y_{s}^{\delta}, Z_{s}^{\delta}\right)-g\left(s, X_{s}^{\star}, Y_{s}^{\star}, Z_{s}^{\star}\right) d s\right.} \\
& +\left(h\left(X_{T}^{\delta}\right)-h\left(X_{T}^{\star}\right)\right) \chi_{\sigma \wedge \tau^{\delta}=T}+a_{\sigma}^{U}\left(X_{\sigma}^{\delta}-X_{\sigma}^{\star}\right) \chi_{\substack{\sigma<\tau^{\delta} \\
\sigma<T}} \\
& \left.+a_{\tau^{\delta}}^{L}\left(X_{\tau^{\delta}}^{\delta}-X_{\tau^{\delta}}^{\star}\right) \chi_{\substack{\tau^{\delta}<\sigma \\
\tau^{\delta}<T}}+\int_{\left[t, \sigma \wedge \tau^{\delta}\right)} a_{s}^{L} d C_{s}^{L} \mid \mathcal{F}_{t}\right] .
\end{aligned}
$$


We will use this representation in the discussion of limit behaviour of $\frac{1}{\delta}\left(Y^{\delta}-Y^{\star}\right)$. As a first step we establish a result on the convergence of $Y^{\delta}$ to $Y^{\star}$.

Proposition 3.10. Assume that (3.10) holds. Then $Y_{t}^{\delta}$ converges to $Y_{t}^{\star} \boldsymbol{P}$-a.s. uniformly in $t$, and further

$$
\begin{aligned}
& \lim _{\delta \searrow 0} E\left[\sup _{t \leq s \leq T}\left|Y_{s}^{\delta}-Y_{s}^{\star}\right|^{2}\right]=0, \\
& \lim _{\delta \searrow 0} E\left[\int_{t}^{T}\left|Z_{s}^{\delta}-Z_{s}^{\star}\right|^{2} d s\right]=0 .
\end{aligned}
$$

The convergence in (3.14) is monotone.

Proof. We know from proposition 3.9 that $\sup _{t \leq s \leq T}\left(Y_{s}^{\delta}-Y_{s}^{\star}\right)$ is nonnegative and decreases as $\delta$ decreases to zero. If we set $A:=\left\{\omega \mid \lim _{\delta \backslash 0}\left(\sup _{t \leq s \leq T} Y_{s}^{\delta}-Y_{s}^{\star}\right)>\right.$ $0\}$ then $\boldsymbol{P}(A)=0$ follows from (3.14).

But (3.14) and (3.15) follow from lemma 2.13 if we can show that - in the notation of that lemma - $\|\boldsymbol{\delta} \xi\|_{2}^{2},\|\boldsymbol{\delta} g\|_{2}^{2}$ and $|\boldsymbol{\delta} C|_{[a, \bar{l}}^{2}$ converge to zero. Hence it suffices to prove convergence of the data in the respective 2-norms.

We first consider the driver and write

$$
\boldsymbol{\delta} g_{s}=g\left(s, X_{s}^{\delta}, Y_{s}^{\star}, Z_{s}^{\star}\right)-g\left(s, X_{s}^{\star}, Y_{s}^{\star}, Z_{s}^{\star}\right) \leq L \cdot \delta \cdot \Gamma_{s}^{u p},
$$

where $L$ denotes a Lipschitz constant of $g$. Hence $\left\|\boldsymbol{\delta} g_{s}\right\|_{2}^{2} \leq L^{2} \cdot \delta^{2} \cdot\left\|\Gamma_{s}^{u p}\right\|_{2}^{2}$ which converges to zero as $\delta \searrow 0$. The same argument can be applied to $\boldsymbol{\delta} \xi=h\left(X_{T}^{\delta}\right)-h\left(X_{T}^{\star}\right)$.

From the definition of the processes $X^{\delta}, X^{\star}$ we have

$$
\begin{aligned}
\left(X_{\tau^{\delta}}^{\delta}-X_{\tau^{\delta}}^{\star}\right)= & \left(X_{\tau^{0}}^{\delta}-X_{\tau^{0}}^{\star}\right)+\int_{\tau^{0}}^{\tau^{\delta}}\left(b\left(s, X_{s}^{\delta}\right)-b\left(s, X_{s}^{\star}\right)\right) d s \\
& +\int_{\tau^{0}}^{\tau^{\delta}} \sigma\left(s,\left(X_{s}^{\delta}-X_{s}^{\star}\right)\right) d W_{s}-\left(C_{\tau^{\delta}}^{L}-C_{\tau^{0}}^{L}\right) .
\end{aligned}
$$

Also recall that $C^{\delta, L}$ is constant on $\left[t, \tau^{0}\right]$. Therefore we can estimate the difference of controls in the following way:

$$
\begin{aligned}
& |\boldsymbol{\delta} C|_{[a, \bar{l}]}^{2}=E\left[\left(\int_{\bar{\imath}} a_{s}^{L} d\left|C^{\delta, L}-C^{L}\right|_{s}\right)^{2}+\left(\int_{\bar{\imath}} a_{s}^{U} d\left|C^{\delta, U}-C^{U}\right|_{s}\right)^{2}\right] \\
& =E\left[\left(\left(a_{\tau^{\delta}}^{L}\left(X_{\tau^{\delta}}^{\delta}-X_{\tau^{\delta}}^{\star}\right)\right) \chi_{\tau^{\delta}<\sigma}+\int_{\left[t, \sigma \wedge \tau^{\delta}\right)} a_{s}^{L} d C_{s}^{L}\right)^{2}\right. \\
& \left.+\left(a_{\sigma}^{U}\left(X_{\sigma}^{\delta}-X_{\sigma}^{\star}\right)\right)^{2} \boldsymbol{\chi}_{\substack{\sigma \leq \tau^{\delta} \\
\sigma<T}}\right] \\
& \leq E\left[\left(\left(a_{\tau^{\delta}}^{L}\left(X_{\tau^{\delta}}^{\delta}-X_{\tau^{\delta}}^{\star}\right)\right)+\int_{\left[\tau^{0}, \tau^{\delta}\right)} a_{s}^{L} d C_{s}^{L}\right)^{2} \chi_{\tau^{0}<\sigma}{ }_{\tau^{0}<T}\right. \\
& \left.+\left(a_{\sigma}^{U}\left(X_{\sigma}^{\delta}-X_{\sigma}^{\star}\right)\right)^{2} \boldsymbol{\chi}_{\substack{\sigma \leq \tau^{\delta} \\
\sigma<T}}\right] \\
& \leq \delta^{2} E\left[3 ( \operatorname { m a x } _ { \tau ^ { 0 } \leq s \leq \tau ^ { \delta } } a _ { s } ^ { L } ) ^ { 2 } \left(\left|\Gamma_{\tau^{0}}^{u p}\right|^{2}+\left(\int_{\tau^{0}}^{\tau^{\delta}} L \Gamma_{s}^{u p} d s\right)^{2}\right.\right. \\
& \left.\left.+\int_{\tau^{0}}^{\tau^{\delta}}\left|\sigma\left(s, \Gamma_{s}^{u p}\right)\right|^{2} d s\right)\right]+\delta^{2} E\left[\left(a_{\sigma}^{U} \Gamma_{\sigma}^{u p}\right)^{2}\right]
\end{aligned}
$$


The second inequality is due to (3.16), where we have used (3.11), (3.7), and Lipschitz continuity of $b$ and linearity of $\sigma$. The last term obviously converges to zero as $\delta \searrow 0$, which completes the proof.

Estimates for the difference quotient We now define processes $\tilde{R}^{u p, \delta}$ and $\tilde{R}^{l o, \delta}$ which converge to the same limiting process $\tilde{R}$ as $\delta \searrow 0$ and serve as majorants and minorants to the difference quotient $\frac{1}{\delta}\left(Y^{\delta}-Y^{\star}\right)$. Thus they help us investigate its limit behaviour. To facilitate the argument we use the translation to a control-free FBSDE of subsection 2.3; we define

$$
\tilde{Y}_{t}^{\delta}:=\frac{1}{\delta}\left(\left(Y_{t}^{\delta}-Y_{t}^{\star}\right)+\int_{\left[0, \sigma \wedge \tau^{\delta} \wedge t\right)} a_{s}^{L} d C_{s}^{L}\right) \quad \text { and } \quad \tilde{Z}_{t}^{\delta}:=\frac{1}{\delta}\left(Z_{t}^{\delta}-Z_{t}^{\star}\right) .
$$

Then $\left(\tilde{Y}^{\delta}, \tilde{Z}^{\delta}\right)$ is solution to a BSDE with data

$$
\begin{aligned}
\xi^{\tilde{Y}}:= & \frac{1}{\delta}\left(h\left(X_{T}^{\delta}\right)-h\left(X_{T}^{\star}\right)\right) \chi_{\sigma \wedge \tau^{\delta}=T}+a_{\sigma}^{U} \frac{1}{\delta}\left(X_{\sigma}^{\delta}-X_{\sigma}^{\star}\right) \chi_{\substack{\sigma<\tau^{\delta} \\
\sigma<T}} \\
+ & a_{\tau^{\delta}}^{L} \frac{1}{\delta}\left(X_{\tau^{\delta}}^{\delta}-X_{\tau^{\delta}}^{\star}\right) \chi_{\tau^{\delta}<\sigma}+\frac{1}{\delta} \int_{\left[0, \sigma \wedge \tau^{\delta}\right)} a_{s}^{L} d C_{s}^{L} \\
g^{\tilde{Y}}(t, y, z):= & \left(\Delta_{x} \tilde{g}_{t} \frac{1}{\delta}\left(X_{t}^{\delta}-X_{t}^{\star}\right)+\Delta_{y} \tilde{g}_{t} \chi_{y \geq 0} \cdot y\right. \\
& \left.-\Delta_{y} \tilde{g}_{t} \frac{1}{\delta} \int_{[0, t)} a_{s}^{L} d C_{s}^{L}\right) \chi_{t \leq \sigma \wedge \tau^{\delta}}+\Delta_{z} \tilde{g}_{t} \cdot z \chi_{t \leq \sigma} \\
C_{t}^{\tilde{Y}}:= & (0,0) .
\end{aligned}
$$

Here $\Delta_{x} \tilde{g}_{t}, \Delta_{y} \tilde{g}_{t}$ and $\Delta_{z} \tilde{g}_{t}$ are defined as:

$$
\begin{aligned}
& \Delta_{x} \tilde{g}_{t}:=\left(g\left(t, X_{t}^{\delta}, Y_{t}^{\star}, Z_{t}^{\star}\right)-g\left(t, X_{t}^{\star}, Y_{t}^{\star}, Z_{t}^{\star}\right)\right) \frac{1}{X_{t}^{\delta}-X_{t}^{\star}} \chi_{X_{t}^{\delta} \neq X_{t}^{\star}}, \\
& \Delta_{y} \tilde{g}_{t}:=\left(g\left(t, X_{t}^{\delta}, Y_{t}^{\delta}, Z_{t}^{\star}\right)-g\left(t, X_{t}^{\delta}, Y_{t}^{\star}, Z_{t}^{\star}\right)\right) \frac{1}{Y_{t}^{\delta}-Y_{t}^{\star}} \chi_{Y_{t}^{\delta} \neq Y_{t}^{\star}}, \\
& \Delta_{z} \tilde{g}_{t}:=\left(g\left(t, X_{t}^{\delta}, Y_{t}^{\delta}, Z_{t}^{\delta}\right)-g\left(t, X_{t}^{\delta}, Y_{t}^{\delta}, Z_{t}^{\star}\right)\right) \frac{1}{Z_{t}^{\delta}-Z_{t}^{\star}} \chi_{Z_{t}^{\delta} \neq Z_{t}^{\star}}
\end{aligned}
$$

Observe that $Y_{t}^{\delta}-Y_{t}^{\star}=0$ for $t>\sigma \wedge \tau^{\delta}$, hence $Y_{t}^{\delta}-Y_{t}^{\star}$ is $\mathcal{F}_{\sigma \wedge \tau^{\delta}}$-measurable. Therefore $Z_{t}^{\delta}-Z_{t}^{\star}=0$ for $t>\sigma \wedge \tau^{\delta}$ and we can in effect extend the $\Delta_{z} \tilde{g}$ component in the definition of $g^{\tilde{Y}}$ from $\left[0, \sigma \wedge \tau^{\delta}\right]$ to $[0, \sigma]$. Observe also that $\tilde{Y}^{\delta} \geq 0$ by (3.1f) and proposition 3.9, and that $\tilde{Y}_{t}^{\delta}=\frac{1}{\delta}\left(Y_{t}^{\delta}-Y_{t}^{\star}\right)$ for $t \leq \sigma \wedge \tau^{0}$. The representation of $\left(\tilde{Y}^{\delta}, \tilde{Z}^{\delta}\right)$ as solution of a BSDE with the data $\left(\xi^{\tilde{Y}}, g^{\tilde{Y}}, C^{\tilde{Y}}\right)$ can be verified directly.

In the following discussion we will assume that (3.1) holds. Therefore we drop the $z$-variable in the notation of partial derivatives of $g$. Observe also that $\Delta_{x} \tilde{g}, \Delta_{y} \tilde{g}$ and $\Delta_{z} \tilde{g}$ do not depend on $Z^{\star}$ and $Z^{\delta}$ in this case.

Now assume $g$ to be partially differentiable and define $\left(\tilde{R}^{u p, \delta}, \tilde{Q}^{u p, \delta}\right)$ as so- 
lution to the BSDE with data

$$
\begin{aligned}
\xi^{u p, \delta}:= & h_{x}\left(X_{T}^{x+\delta, C^{U}, 0}\right) \Gamma_{T}^{x+\delta, C^{U}, 0} \chi_{\sigma \wedge \tau^{\delta}=T}+a_{\sigma}^{U} \Gamma_{\sigma}^{x+\delta, C^{U}, 0} \chi_{\sigma \leq \tau^{\delta}} \\
+ & \left(\max _{\tau^{0} \leq s \leq \tau^{\delta}} a_{s}^{L}\right)\left(\sup _{\tau^{0} \leq s \leq \tau^{\delta}} \Gamma_{s}^{x+\delta, C^{U}, 0}\right)^{2}\left(\inf _{\tau^{0} \leq s \leq \tau^{\delta}} \Gamma_{s}^{x, C^{U}, 0}\right)^{-1} \chi_{\tau^{0}<\sigma} \tau^{0}<T \\
g^{u p, \delta}(t, y, z):= & \left(g_{x}\left(t, X_{t}^{\delta}, Y_{t}^{\star}\right) \Gamma_{t}^{x+\delta, C^{U}, 0}+g_{y}\left(t, X_{t}^{\delta}, Y_{t}^{\delta}\right) \chi_{y \geq 0} \chi_{A_{g, \delta}^{+}} \cdot y\right. \\
& \left.+L\left(\max _{\tau^{0} \leq s \leq \tau^{\delta} \wedge t} a_{s}^{L}\right) \overline{C_{t}^{L, \delta}}\right) \chi_{t \leq \sigma \wedge \tau^{\delta}}+g_{z}(t) \cdot z \chi_{t \leq \sigma} \\
C_{t}^{u p, \delta}:= & (0,0) .
\end{aligned}
$$

Similarly we let $\left(\tilde{R}^{l o, \delta}, \tilde{Q}^{l o, \delta}\right)$ the solution to the BSDE with data

$$
\begin{aligned}
\xi^{l o, \delta}:= & h_{x}\left(X_{T}^{x, C^{U}, 0}\right) \Gamma_{T}^{x, C^{U}, 0} \chi_{\sigma \wedge \tau^{0}=T}+a_{\sigma}^{U} \Gamma_{\sigma}^{x, C^{U}, 0} \chi_{\substack{\sigma<\tau^{0} \\
\sigma<T}}+\left(\min _{\tau^{0} \leq s \leq \tau^{\delta}} a_{s}^{L}\right)\left(\inf _{\tau^{0} \leq s \leq \tau^{\delta}} \Gamma_{s}^{x, C^{U}, 0}\right)^{2}\left(\sup _{\tau^{0} \leq s \leq \tau^{\delta}} \Gamma_{s}^{x+\delta, C^{U}, 0}\right)^{-1} \chi_{\tau^{\delta}<\sigma}< \\
g^{l o, \delta}(t, y, z):= & g_{x}\left(t, X_{t}^{\star}, Y_{t}^{\star}\right) \Gamma_{t}^{x, C^{U}, 0} \chi_{t \leq \sigma \wedge \tau^{0}}+\left(g_{y}\left(t, X_{t}^{\star}, Y_{t}^{\star}\right) \chi_{y \geq 0} \chi_{A_{g, \delta}^{-}} \cdot y\right. \\
& \left.-L\left(\max _{\tau^{0} \leq s \leq \tau^{\delta} \wedge t} a_{s}^{L}\right) \overline{C_{t}^{L, \delta}}\right) \chi_{t \leq \sigma \wedge \tau^{\delta}}+g_{z}(t) \cdot z \chi_{t \leq \sigma} \\
C_{t}^{l o, \delta}:= & (0,0) .
\end{aligned}
$$

Recall the definition of $\overline{C_{t}^{L, \delta}}$ in lemma 3.7. $L$ again denotes a Lipschitz constant of $g$.

We need $A_{g, \delta}^{+}$and $A_{g, \delta}^{-}$to eliminate the negative and positive parts in the $g_{y} \cdot y$-terms for $t>\sigma \wedge \tau^{0}$. The reason is that $\chi_{t \leq \tau^{\delta}}$ decreases to $\chi_{t \leq \tau^{0}}$ in $\delta$, so we have to add a condition that ensures the required monotonicity of the data. More specific, we define

$$
\begin{aligned}
& A_{g, \delta}^{+}:=\left\{t \leq \sigma \wedge \tau^{0}\right\} \cup\left\{g_{y}\left(t, X_{t}^{\delta}, Y_{t}^{\delta}\right) \geq 0\right\} \\
& A_{g, \delta}^{-}:=\left\{t \leq \sigma \wedge \tau^{0}\right\} \cup\left\{g_{y}\left(t, X_{t}^{\star}, Y_{t}^{\star}\right) \leq 0\right\}
\end{aligned}
$$

We also use the non-standard convention $\max _{\tau^{0}<s<\tau^{\delta} \wedge t} a_{s}^{L}=0$ for $t<\tau^{0}$.

We are now in a position to formulate the estimate.

Lemma 3.11. Assume that (2.31) and (3.1) hold. Then $\tilde{R}^{u p, \delta}, \tilde{Y}^{\delta}, \tilde{R}^{l o, \delta}$ satisfy

$$
\tilde{R}_{t}^{l o, \delta} \leq \tilde{Y}_{t}^{\delta} \leq \tilde{R}_{t}^{u p, \delta} \quad \forall t \in \bar{\imath} \boldsymbol{P} \text {-a.s. }
$$

Further, $\tilde{R}^{u p, \delta}$ decreases and $\tilde{R}^{\text {lo, } \delta}$ increases as $\delta \searrow 0$ to the same limiting process $\tilde{R}$ defined below, in $L_{\mathcal{F}}^{2}(0, T ; \mathbb{R})$ and for all $t \in \bar{\imath} \boldsymbol{P}$-a.s.

Proof. To apply the comparison theorem 2.14 with supplementary condition $(2.27 \mathrm{c})$ to $\tilde{R}^{u p, \delta}, \tilde{Y}^{\delta}, \tilde{R}^{l o, \delta}$ we have to estimate the drivers and terminal conditions of the three processes. 
Consider the drivers first. Their components satisfy

$$
\begin{gathered}
g_{x}\left(t, X_{t}^{\star}, Y_{t}^{\star}\right) \Gamma_{t}^{x, C^{U}, 0} \chi_{t \leq \sigma \wedge \tau^{0}} \leq \Delta_{x} \tilde{g}_{t} \frac{1}{\delta}\left(X_{t}^{\delta}-X_{t}^{\star}\right) \chi_{t \leq \sigma \wedge \tau^{\delta}} \\
\leq g_{x}\left(t, X_{t}^{\delta}, Y_{t}^{\star}\right) \Gamma_{t}^{x+\delta, C^{U}, 0} \chi_{t \leq \sigma \wedge \tau^{\delta}}, \\
\begin{array}{c}
g_{y}\left(t, X_{t}^{\star}, Y_{t}^{\star}\right) \frac{1}{\delta}\left(Y_{t}^{\delta}-Y_{t}^{\star}\right) \chi_{A_{g, \delta}^{-}} \leq \Delta_{y} \tilde{g}_{t} \frac{1}{\delta}\left(Y_{t}^{\delta}-Y_{t}^{\star}\right) \\
\leq g_{y}\left(t, X_{t}^{\delta}, Y_{t}^{\delta}\right) \frac{1}{\delta}\left(Y_{t}^{\delta}-Y_{t}^{\star}\right) \chi_{A_{g, \delta}^{+},} \\
g_{y}\left(t, X_{t}^{\star}, Y_{t}^{\star}\right) \frac{1}{\delta} \int_{\left[\tau^{0}, \tau^{\delta} \wedge t\right)} a_{s}^{L} d C_{s}^{L}-L\left(_{\tau^{0} \leq s \leq \tau^{\delta} \wedge t} a_{s}^{L}\right) \overline{C_{t}^{L, \delta}} \\
\leq \Delta_{y} \tilde{g}_{t} \frac{1}{\delta} \int_{\left[\tau^{0}, \tau^{\delta} \wedge t\right)} a_{s}^{L} d C_{s}^{L}-\Delta_{y} \tilde{g}_{t} \frac{1}{\delta} \int_{\left[\tau^{0}, \tau^{\delta} \wedge t\right)} a_{s}^{L} d C_{s}^{L} \quad(\text { which equals zero }) \\
\leq g_{y}\left(t, X_{t}^{\delta}, Y_{t}^{\delta}\right) \frac{1}{\delta} \int_{\left[\tau^{0}, \tau^{\delta} \wedge t\right)} a_{s}^{L} d C_{s}^{L}+L\left(\max _{\tau^{0} \leq s \leq \tau^{\delta} \wedge t} a_{s}^{L}\right) \overline{C_{t}^{L, \delta}} \\
g_{z}(t) \frac{1}{\delta}\left(Z_{t}^{\delta}-Z_{t}^{\star}\right)=\Delta_{z} \tilde{g}_{t} \frac{1}{\delta}\left(Z_{t}^{\delta}-Z_{t}^{\star}\right)
\end{array}
\end{gathered}
$$

The estimates for $\Delta_{x} \tilde{g}$ follow from (3.1c), (2.31d) and (3.9). For the estimates of $\Delta_{y} \tilde{g}$ use $(2.31 \mathrm{~d}),(3.1 \mathrm{c})$, proposition 3.9 and the definition of $A_{g, \delta}^{ \pm}$.

For the third line estimate of the $L a^{L}$-term recall Lipschitz-continuity of $g$ and lemma 3.7. As $g_{z}$ is independent of $(x, y, z)$ and $\tau^{\delta} \geq \tau^{0}$ we can summarize this as

$$
g^{l o, \delta}\left(t, \tilde{Y}^{\delta}, \tilde{Z}^{\delta}\right) \leq g^{\tilde{Y}}\left(t, \tilde{Y}^{\delta}, \tilde{Z}^{\delta}\right) \leq g^{u p, \delta}\left(t, \tilde{Y}^{\delta}, \tilde{Z}^{\delta}\right) .
$$

In a similar way we can estimate the terms in $h_{x}, a^{U}$ and $h$ respectively in the definitions of $\xi^{l o, \delta}, \xi^{\tilde{Y}}$ and $\xi^{u p, \delta}$ by (2.31c), (3.1b) and (3.9). For the terms in $a^{L}$ observe that, by lemma 3.7 and (2.38), (3.12)

$$
\begin{aligned}
& a_{\tau^{\delta}}^{L} \frac{1}{\delta}\left(X_{\tau^{\delta}}^{\delta}-X_{\tau^{\delta}}^{\star}\right) \chi_{\tau^{\delta}<\sigma}+\frac{1}{\delta} \int_{\left[\sigma \wedge \tau^{0}, \sigma \wedge \tau^{\delta}\right)} a_{s}^{L} d C_{s}^{L} \\
& \leq \frac{1}{\delta}\left(\max _{\tau^{0} \leq s \leq \tau^{\delta}} a_{s}^{L}\right)\left(X_{\tau^{\delta}}^{\delta}-X_{\tau^{\delta}}^{\star}+\left(X_{\tau^{\delta}}^{\star}-X_{\tau^{\delta}}^{x, C^{U}, 0}\right)\left(\Gamma_{\tau^{\delta}}^{\tilde{\Theta}}\right)^{-1}\left(\sup _{\tau^{0} \leq s \leq \tau^{\delta}} \Gamma_{s}^{\tilde{\Theta}}\right)\right) \chi_{\tau_{\tau^{0}<\sigma}<T} \\
& \quad \leq\left(\max _{\tau^{0} \leq s \leq \tau^{\delta}} a_{s}^{L}\right)\left(\sup _{\tau^{0} \leq s \leq \tau^{\delta}} \Gamma_{s}^{x+\delta, C^{U}, 0}\right)^{2}\left(\inf _{\tau^{0} \leq s \leq \tau^{\delta}} \Gamma_{s}^{x, C^{U}, 0}\right)^{-1} \chi_{\tau^{0}<\sigma} . \\
& \tau^{0}<T
\end{aligned}
$$

Similarly we can estimate the $a^{L}$-term in $\xi^{l o, \delta}$ :

$$
\begin{aligned}
\left(\min _{\tau^{0} \leq s \leq \tau^{\delta}} a_{s}^{L}\right)\left(\inf _{\tau^{0} \leq s \leq \tau^{\delta}} \Gamma_{s}^{x, C^{U}, 0}\right)^{2}\left(\sup _{\tau^{0} \leq s \leq \tau^{\delta}} \Gamma_{s}^{x+\delta, C^{U}, 0}\right)^{-1} \chi_{\tau^{\delta}<\sigma} & \\
& \leq a_{\tau^{\delta}}^{L} \frac{1}{\delta}\left(X_{\tau^{\delta}}^{\delta}-X_{\tau^{\delta}}^{\star}\right) \chi_{\tau^{\delta}<T}+\frac{1}{\delta} \int_{\substack{\tau^{\delta}<T \\
\left[\sigma \wedge \tau^{0}, \sigma \wedge \tau^{\delta}\right)}} a_{s}^{L} d C_{s}^{L}
\end{aligned}
$$

Hence $\xi^{l o, \delta} \leq \xi^{\delta} \leq \xi^{u p, \delta}$. An application of the comparison theorem 2.14 and (2.27c) completes the proof of (3.17).

Let us now prove monotony of $\tilde{R}^{l o, \delta}$ and $\tilde{R}^{u p, \delta}$ in $\delta$. We show that the data are monotone in $\delta$ so that we can again apply the comparison theorem 2.14. 
Consider the terminal values first. The summands in $\xi^{u p, \delta}$ are nonnegative. The terms in $h_{x}$ and $a^{U}$ decrease with $\delta$ by (3.1b), (3.1f), (2.40) and $\tau^{\delta} \searrow \tau^{0}$. The third term decreases to $a_{\tau^{0}}^{L} \Gamma_{\tau^{0}}^{x, C^{U}, 0}$, as $a^{L}$ and $\Gamma$ are continuous.

The terms in $h_{x}$ and $a^{U}$ of $\xi^{\tau^{o, \delta}}$ are independent of $\delta$. The $a^{L}$-term increases as $\delta \searrow 0$, as $\sup _{\tau^{0} \leq s \leq \tau^{\delta}} \Gamma_{s}^{x+\delta, C^{U}, C^{L}}$ decreases and $\boldsymbol{\chi}_{\tau^{\delta}<\sigma, \tau^{\delta}<T}$ increases. Hence $\xi^{u p, \delta}$ and $\xi^{l o, \delta}$ decrease and increase respectively to $\tilde{\xi}$, where

$$
\tilde{\xi}:=h_{x}\left(X_{T}^{x, C^{U}, 0}\right) \Gamma_{T}^{x, C^{U}, 0} \chi_{\sigma \wedge \tau^{0}=T}+a_{\sigma}^{U} \Gamma_{\sigma}^{x, C^{U}, 0} \chi_{\sigma \leq \tau^{0}}+a_{\tau^{0}}^{L} \Gamma_{\tau^{0}}^{x, C^{U}, 0}{ }_{\substack{\tau^{0}<T \\ \tau^{0}<T}}
$$

Next we show that the drivers $g^{u p, \delta}$ and $g^{l o, \delta}$ decrease and increase respectively and, for $t \neq \sigma \wedge \tau^{0}$ converge to $\tilde{g}$ defined by

$$
\begin{aligned}
\tilde{g}(t, y, z):= & \left(g_{x}\left(t, X_{t}^{\star}, Y_{t}^{\star}\right) \Gamma_{t}^{x, C^{U}, 0}+g_{y}\left(t, X_{t}^{\star}, Y_{t}^{\star}\right) \chi_{y \geq 0} \cdot y\right) \chi_{t \leq \sigma \wedge \tau^{0}} \\
& +g_{z}(t) \cdot z \boldsymbol{\chi}_{t \leq \sigma} .
\end{aligned}
$$

Convergence and monotony of the $g_{x}$-term of $g^{u p, \delta}$ is obvious; for $g^{l o, \delta}$ it is trivial. The terms in $\pm L\left(\max _{\tau^{0}<s<\tau^{\delta} \wedge t} a_{s}^{L}\right)$ equal zero for $t<\sigma \wedge \tau^{0}$; they are decreasing in absolute value. As $\chi_{\tau^{0} \leq t \leq \sigma \wedge \tau^{\delta}}$ converges to zero, both terms decrease and increase respectively to zero for $t \neq \sigma \wedge \tau^{0}$.

For $t \leq \sigma \wedge \tau^{0}$ the $g_{y} \cdot y$-term in $g^{u p, \delta}$ decreases by (3.1d), monotony of $X^{\delta}$ in $\delta$ and proposition 3.9. For $t>\sigma \wedge \tau^{0}$ it decreases and is nonnegative thanks to the definition of $A_{g, \delta}^{+}$. As $\chi_{t \leq \sigma \wedge \tau^{\delta}}$ decreases to zero the full $g_{y} \cdot y$-component also decreases to zero.

Convergence of the $g_{y} \cdot y$-term in $g^{l o, \delta}$ for $t \leq \sigma \wedge \tau^{0}$ is trivial. For $t>\sigma \wedge \tau^{0}$ we are restricted to the negative values by definition of $A_{g, \delta}^{-}$, and as $\chi_{t \leq \sigma \wedge \tau^{\delta}}$ decreases to zero the $g_{y} \cdot y$-term increases to zero.

The $g_{z}$-term remains unaffected by (3.1e). So $g^{l o, \delta} \nearrow \tilde{g}$ and $g^{u p, \delta} \searrow \tilde{g}$ as $\delta \searrow 0$ for $t \neq \sigma \wedge \tau^{0}$.

Let $(\tilde{R}, \tilde{Q})$ the solution to the $\operatorname{BSDE}$ with data $(\tilde{\xi}, \tilde{g}, 0)$. $\tilde{R}$ will serve as limiting process for $\tilde{R}^{u p, \delta}$ and $\tilde{R}^{l o, \delta}$. By the comparison theorem, left continuity of the prcesses involved and the above discussion of the data $\left(\xi^{u p, \delta}, g^{u p, \delta}\right)$ and $\left(\xi^{l o, \delta}, g^{l o, \delta}\right)$ we have

$$
\tilde{R}_{t}^{l o, \delta} \leq \tilde{R}_{t} \leq \tilde{R}_{t}^{u p, \delta} \quad \forall t \in \bar{\imath} \boldsymbol{P} \text {-a.s. }
$$

and $\tilde{R}_{t}-\tilde{R}_{t}^{l o, \delta}$ and $\tilde{R}_{t}^{u p, \delta}-\tilde{R}_{t}$ decrease as $\delta \searrow 0$.

Furthermore, the differences of the data and hence the processes $\tilde{R}_{t}-\tilde{R}_{t}^{l o, \delta}$ and $\tilde{R}_{t}^{u p, \delta}-\tilde{R}_{t}$ decrease to zero in $L_{\mathcal{F}}^{2}(0, T ; \mathbb{R})$. To be precise, set

$$
\begin{aligned}
\left\|\boldsymbol{\delta} g^{u p, \delta}\right\|_{2}^{2} & :=E\left[\int_{0}^{\sigma \wedge \tau^{\delta}}\left|\tilde{g}\left(s, \tilde{R}_{s}, \tilde{Q}_{s}\right)-g^{u p, \delta}\left(s, \tilde{R}_{s}, \tilde{Q}_{s}\right)\right|^{2} d s\right], \\
\left\|\boldsymbol{\delta} g^{l o, \delta}\right\|_{2}^{2} & :=E\left[\int_{0}^{\sigma \wedge \tau^{\delta}}\left|\tilde{g}\left(s, \tilde{R}_{s}, \tilde{Q}_{s}\right)-g^{l o, \delta}\left(s, \tilde{R}_{s}, \tilde{Q}_{s}\right)\right|^{2} d s\right], \\
\left\|\boldsymbol{\delta} \xi^{u p, \delta}\right\|_{2}^{2} & :=E\left[\left|\tilde{\xi}-\xi^{u p, \delta}\right|^{2}\right], \\
\left\|\boldsymbol{\delta} \xi^{l o, \delta}\right\|_{2}^{2} & :=E\left[\left|\tilde{\xi}-\xi^{l o, \delta}\right|^{2}\right],
\end{aligned}
$$


which converge to zero as $\delta \searrow 0$ by the dominated convergence theorem. The a priori estimates in proposition 2.13 show that

$$
\lim _{\delta \searrow 0} E\left[\sup _{0 \leq s \leq T}\left|\tilde{R}_{s}^{u p, \delta}-\tilde{R}_{s}\right|^{2}\right]=\lim _{\delta \searrow 0} E\left[\sup _{0 \leq s \leq T}\left|\tilde{R}_{s}^{l o, \delta}-\tilde{R}_{s}\right|^{2}\right]=0 .
$$

By monotony of $\tilde{R}^{l o, \delta}$ and $\tilde{R}^{u p, \delta}$ these converge also $\boldsymbol{P}$-a.s. to uniformly in $t$ to $\tilde{R}$. As $T \in \mathbb{R}_{>0}$ is bounded, $\tilde{R}^{l o, \delta}$ and $\tilde{R}^{u p, \delta}$ converge to $\tilde{R}$ in $L_{\mathcal{F}}^{2}(0, T ; \mathbb{R})$.

Recall the payoff $R$ of the associated Dynkin game in definition 2.5. Now define the data

$$
\begin{aligned}
\xi^{R}:= & \left(h_{x}\left(X_{T}^{x, 0,0}\right) \chi_{\sigma \wedge \tau^{0}=T}+a_{\sigma}^{U} \chi_{\sigma \leq \tau^{0}}+a_{\tau^{0}}^{L} \chi_{\tau^{0}<\sigma}\right) \Gamma_{\tau^{0}<T}^{x} \underset{\sigma \wedge \tau^{0} \wedge T}{ } \\
g^{R}(t, y, z):= & \left(g_{x}\left(t, X_{t}^{x, 0,0}, Y_{t}^{\star}\right) \Gamma_{t}^{x}+g_{y}\left(t, X_{t}^{x, 0,0}, Y_{t}^{\star}\right) \cdot y\right) \chi_{t \leq \sigma \wedge \tau^{0}} \\
& +g_{z}(t) \cdot z \chi_{t \leq \sigma} \\
\text { and } C^{R}:= & 0
\end{aligned}
$$

Then the solution $(R, Q)$ of the BSDE with data $\left(\xi^{R}, g^{R}, a, 0\right)$ is the payoff defined in (2.5) and has the representation:

$$
\begin{aligned}
& R_{t}^{x}\left(\sigma, \tau^{0}\right)=E\left[\int_{t}^{\sigma \wedge \tau^{0}}\left\langle D g\left(s, X_{s}^{x, 0,0}, Y_{s}^{\star}\right),\left(\Gamma_{s}^{x}, R_{s}, Q_{s}\right)\right\rangle d s\right. \\
& \left.+h_{x}\left(X_{T}^{x, 0,0}\right) \Gamma_{T}^{x} \chi_{\sigma \wedge \tau^{0}=T}+a_{\sigma}^{U} \Gamma_{\sigma}^{x} \chi_{\substack{\sigma<\tau^{0} \\
\sigma<T}}+a_{\tau^{0}}^{L} \Gamma_{\tau^{0}}^{x} \chi_{\tau^{0}<\sigma}<\mid \mathcal{F}_{t}\right]
\end{aligned}
$$

Lemma 3.12. Assume that (2.31) and (3.1) hold. Then

$$
\lim _{\delta \searrow 0} \frac{1}{\delta}\left(Y_{t}^{\delta}-Y_{t}^{\star}\right) \leq R_{t}^{x}\left(\sigma, \tau^{0}\right)
$$

holds $\boldsymbol{P}$-a.s. for all $t \leq \sigma \wedge \tau^{0}$.

Proof. We use the notation of lemma 3.11. From the construction of $\tilde{Y}^{\delta}$ we have $\tilde{Y}_{t}^{\delta}=\frac{1}{\delta}\left(Y_{t}^{\delta}-Y_{t}^{\star}\right)$ for $t \leq \sigma \wedge \tau^{0}$. Hence by $(3.17)$

$$
\tilde{R}_{t}^{l o, \delta} \leq \frac{1}{\delta}\left(Y_{t}^{\delta}-Y_{t}^{\star}\right) \leq \tilde{R}_{t}^{u p, \delta} \quad \text { for } t \leq \sigma \wedge \tau^{0} \boldsymbol{P} \text {-a.s. }
$$

As $\tilde{R}^{l o, \delta}$ and $\tilde{R}^{u p, \delta}$ converge to $\tilde{R}$, so does $\frac{1}{\delta}\left(Y^{\delta}-Y^{\star}\right)$. Hence the limit in (3.20) exists and it suffices to prove

$$
\tilde{R}_{t} \leq R_{t}^{x}\left(\sigma, \tau^{0}\right) \quad \text { for } t \leq \sigma \wedge \tau^{0} \boldsymbol{P} \text {-a.s. }
$$

First observe that $\tilde{R}$ remains unchanged if we restrict the $g_{z}$-term in the definition of $\tilde{g}$ to $\left[0, \sigma \wedge \tau^{0}\right]$. This follows from the fact that $\tilde{R}_{t}$ is $\mathcal{F}_{\sigma \wedge \tau^{0}}$-measurable for $t>\sigma \wedge \tau^{0}$, hence $\tilde{Q}_{t}=0$ on $\left(\sigma \wedge \tau^{0}, T\right]$.

Recall that $X_{t}^{\star}=X_{t}^{x, C^{U}, 0}$ for $t \leq \tau^{0}$ by definition and that $X^{x, C^{U}, 0} \leq X^{x, 0,0}$ and $\Gamma^{x, C^{U}, 0} \leq \Gamma^{x, 0,0}$ by $(2.31 \mathrm{a})$ and the comparison theorem 2.8 .

Hence $\tilde{g}(t, y, z) \leq g^{R}(t, y, z)$ is a consequence of (2.31d), (3.1c). Using (2.31c), (3.1b) and (3.1f) we deduce $\tilde{\xi} \leq \xi^{R}$. Hence the assumptions of the comparison theorem are satisfied, which completes the proof. 
Let us now consider the case where $C=\left(C^{U},-C^{L}\right)$ is optimal for the problem starting in $\left(t_{0}, x\right)$, i.e. the value $V_{t}\left(t_{0}, x\right)$ is the state process of a controlled BSDE:

$$
V_{t}\left(t_{0}, x\right)=Y_{t}^{\star}=Y_{t}^{t_{0}, x, C} \quad \forall t \geq t_{0} .
$$

Proposition 3.13. Assume that (2.31) and (3.1) hold that there exists an optimal control $\left(C^{U}, C^{L}\right)$ for the control problem 2.2 in $\left(t_{0}, x\right)$. Let $V_{t}\left(t_{0}, x\right)$ its value. Then the upper right Dini derivative of $V$ with respect to the initial condition satisfies

$$
\Delta^{+} V_{t}\left(t_{0}, x\right) \leq u_{t}^{-}\left(t_{0}, x\right) \quad \forall t \geq t_{0} \boldsymbol{P} \text {-a.s. }
$$

Proof. By optimality of $C$ in $\left(t_{0}, x\right)$ and lemma 3.12 we have

$$
\limsup _{\delta \searrow 0} \frac{1}{\delta}\left(V_{t}\left(t_{0}, x+\delta\right)-V_{t}\left(t_{0}, x\right)\right) \leq \limsup _{\delta \searrow 0} \frac{1}{\delta}\left(Y_{t}^{\delta}-Y_{t}^{\star}\right) \leq R_{t}^{X}\left(\sigma, \tau^{0}\right)
$$

As $\sigma \in \mathcal{T}_{t_{0}}$ is arbitrary this gives

$$
\Delta^{+} V_{t}\left(t_{0}, x\right) \leq \operatorname{essinf}_{\sigma \in \mathcal{T}_{t_{0}}} R_{t}\left(\sigma, \tau^{0}\right) \leq u_{t}^{-}\left(t_{0}, x\right) \quad \boldsymbol{P} \text {-a.s. }
$$

\subsection{Lower left Dini derivative}

The construction of an estimate for the lower left dini derivative of $V$ is essentially the same as that in section 3.1. This is intuitively obvious if we consider $-X$ instead of $X$ as controlled forward process, and all proofs relying on the set of conditions in (3.10) translate one to one to the new situation. For example, $C^{U}$ and $C^{L}$ just change their roles. We therefore only line out the argument by listing the statements.

However, for our investigation of the difference quotient the transformation $X \mapsto-X$ cannot be applied as it does not preserve convexity of the data. So we give the definitions of upper and lower bounds and limiting process for the difference quotient in detail.

Construction of the tracking process from below We construct a process $X^{-\delta}$ that tracks a given controlled process $X^{\star}=X^{t_{0}, x, C^{U}, C^{L}}$, starting at a distance $\delta$ below $x$ and jumping on $X^{\star}$ some time afterwards.

Let $\tau \in \mathcal{T}_{t_{0}}$ arbitrary and set the crossing time

$$
\sigma^{\delta}:=\inf \left\{t \geq t_{0} \mid X_{t}^{x-\delta, 0, C^{L}} \geq X_{t}^{x, C^{U}, C^{L}}\right\}
$$

The tracking process $X^{-\delta}$ for the starting point $x-\delta$ is defined as

$$
X_{t}^{-\delta}:= \begin{cases}X_{t}^{t_{0}, x-\delta, 0, C^{L}} & t \leq \sigma^{\delta} \wedge \tau \\ X_{t}^{t_{0}, x, C^{U}, C^{L}} & t>\sigma^{\delta} \wedge \tau .\end{cases}
$$

It has parrallel lower and no upper control as long as either $\tau$ occurs or $X^{\star}$ crosses its path. 
We again suppress dependence on $t_{0}$. To obtain $X^{-\delta}$ as controlled process define $C^{-\delta}=\left(C^{-\delta, U},-C^{-\delta, L}\right)$ by

$$
\begin{aligned}
& C_{t}^{-\delta, U}:= \begin{cases}0 & t \leq \sigma^{\delta} \wedge \tau \\
C_{t}^{U}-C_{\sigma^{\delta}}^{U}-\left(X_{\sigma^{\delta}}^{x, C^{U}, C^{L}}-X_{\sigma^{\delta}}^{x-\delta, 0, C^{L}}\right) & t>\sigma^{\delta}, \sigma^{\delta} \leq \tau \\
C_{t}^{U}-C_{\tau}^{U} & t>\tau, \sigma^{\delta}>\tau\end{cases} \\
& C_{t}^{-\delta, L}:= \begin{cases}C_{t}^{L} & t \leq \tau \\
C_{t}^{L} & t>\tau, \sigma^{\delta} \leq \tau \\
C_{t}^{L}+\left(X_{\tau}^{x, C^{U}, C^{L}}-X_{\tau}^{x-\delta, 0, C^{L}}\right) & t>\tau, \sigma^{\delta}>\tau\end{cases}
\end{aligned}
$$

From the definition of $\sigma^{\delta}$ we have for $t>\sigma^{\delta}, \sigma^{\delta} \leq \tau$ :

$$
\begin{aligned}
0 \leq C_{t}^{U}-C_{\sigma^{\delta}+}^{U}+X_{\sigma^{\delta}+}^{x-\delta, 0, C^{L}}-X_{\sigma^{\delta}+}^{x, C^{U}, C^{L}} & \\
= & C_{t}^{U}-C_{\sigma^{\delta}+}^{U}+X_{\sigma^{\delta}}^{x-\delta, 0, C^{L}}+\left(C_{\sigma^{\delta}+}^{L}-C_{\sigma^{\delta}}^{L}\right) \\
& \quad-\left(X_{\sigma^{\delta}}^{x, C^{U}, C^{L}}-\left(C_{\sigma^{\delta}+}^{U}-C_{\sigma^{\delta}}^{U}\right)+\left(C_{\sigma^{\delta}+}^{L}-C_{\sigma^{\delta}}^{L}\right)\right) \\
& =C_{t}^{U}-C_{\sigma^{\delta}}^{U}-\left(X_{\sigma^{\delta}}^{x, C^{U}, C^{L}}-X_{\sigma^{\delta}}^{x-\delta, 0, C^{L}}\right)
\end{aligned}
$$

so $C^{-\delta, U}$ is increasing, hence $C^{-\delta} \in \mathcal{A}$. In addition to the notation of $X^{\star}, Y^{\star}$ and $Z^{\star}$ in section 3.1 we set

$$
Y_{t}^{-\delta}:=Y_{t}^{x-\delta, C^{-\delta, U}, C^{-\delta, L}}, \quad Z_{t}^{-\delta}:=Z_{t}^{x-\delta, C^{-\delta, U}, C^{-\delta, L}}
$$

The following statements and their proofs are parallel to propositions 3.3, $3.9,3.10$ and lemmata 3.6, 3.7. Instead of a line-by-line imitation we could use a transformation

$$
\begin{aligned}
\hat{b}(t, x) & :=-b(t,-x), & \dot{\sigma}(t, x) & :=\sigma(t, x), \\
\hat{h}(x) & :=-h(-x), & \dot{g}(t, x, y, z) & :=-g(t,-x,-y,-z)
\end{aligned}
$$

by which

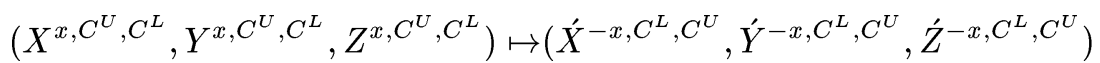

$$
\begin{aligned}
& =\left(-X^{x, C^{U} \cdot C^{L}},-Y^{x, C^{U}, C^{L}},-Z^{x, C^{U}, C^{L}}\right) .
\end{aligned}
$$

and apply the results of section 3.1. This is essentially due to the fact that above transformation does not affect validity of (3.10).

Proposition 3.14. Let $C^{-\delta}$ as defined in (3.25) and assume (3.10) holds. Then $X^{x-\delta, C^{-\delta, U}, C^{-\delta, L}}=X^{-\delta}$ and

$$
0 \leq X_{t}^{x, C^{U}, C^{L}}-X_{t}^{x-\delta, C^{-\delta, U}, C^{-\delta, L}} \leq X_{t}^{x, 0, C^{L}}-X_{t}^{x-\delta, 0, C^{L}} .
$$

Further $Y_{t}^{x-\delta, C^{-\delta, U}, C^{-\delta, L}}=Y_{t}^{x, C^{U}, C^{L}}$ and $Z_{t}^{x-\delta, C^{-\delta, U}, C^{-\delta, L}}=Z_{t}^{x, C^{U}, C^{L}}$ for $\sigma^{\delta} \wedge \tau<t<T$.

$X_{t}^{x-\delta, C^{\bar{U}}, C^{L}}$ increases as $\delta$ decreases and $X^{-\delta}$ increases to $X^{\star}$. 
A bound for $C^{U}$ Define the first action time $\sigma^{0}$ of $C^{U}$ by

$$
\sigma^{0}:=\inf \left\{t \geq t_{0} \mid X_{t}^{x, 0, C^{L}}>X_{t}^{\star}\right\}=\inf \left\{t \geq t_{0} \mid C_{t}^{U}>C_{t_{0}}^{U}\right\}
$$

and observe that $\sigma^{\delta} \searrow \sigma^{0} \boldsymbol{P}$-a.s. With $\hat{\Theta}$ defined suitably and $\Gamma^{\hat{\Theta}}$ the solution of the linear SDE

$$
d \Gamma_{t}^{\hat{\Theta}}=b_{x}\left(t, \hat{\Theta}_{t}\right) \Gamma_{t}^{\hat{\Theta}} d t+\sigma\left(t, \Gamma_{t}^{\hat{\Theta}}\right) d W_{t}, \quad \hat{X}_{0}=1,
$$

the representation

$$
\begin{aligned}
X_{t}^{x, 0, C^{L}}-X_{t}^{\star}= & \int_{0}^{t} b_{x}\left(s, \hat{\Theta}_{s}\right)\left(X_{s}^{x, 0, C^{L}}-X_{s}^{\star}\right) d s+C_{t}^{U} \\
& +\int_{0}^{t} \sigma\left(s, X_{s}^{x, 0, C^{L}}-X_{s}^{\star}\right) d W_{s}=\int_{\sigma^{0}}^{\sigma^{0} \vee t} \Gamma_{t}^{\hat{\Theta}}\left(\Gamma_{s}^{\hat{\Theta}}\right)^{-1} d C_{s}^{U}
\end{aligned}
$$

leads to the analogue of Lemma 3.7:

Lemma 3.15. Let $\sigma^{0}$ and $\sigma^{\delta}$ as defined in (3.27) and (3.23), and assume (3.10) holds. Then $C_{t}^{U}$ has $\boldsymbol{P}$-a.s. upper and lower bounds for $\sigma^{0}<t \leq \sigma^{\delta}$ :

$$
\left(X_{t}^{x, 0, C^{L}}-X_{t}^{\star}\right) \frac{1}{\Gamma_{t}^{\hat{\Theta}}}\left(\inf _{\sigma^{0}<s \leq t} \Gamma_{s}^{\hat{\Theta}}\right) \leq C_{t}^{U} \leq\left(X_{t}^{x, 0, C^{L}}-X_{t}^{\star}\right) \frac{1}{\Gamma_{t}^{\hat{\Theta}}}\left(\sup _{\sigma^{0}<s \leq t} \Gamma_{s}^{\hat{\Theta}}\right) .
$$

Thus $C^{U}$ satisfies

$$
C_{t}^{U} \leq \delta \Gamma_{t}^{u p}\left(\Gamma_{t}^{l o}\right)^{-1}\left(\sup _{\sigma^{0} \leq s \leq t} \Gamma_{s}^{u p}\right)=: \delta \cdot \overline{C_{t}^{U,-\delta}} \quad \text { for } t \leq \sigma^{\delta} \boldsymbol{P} \text {-a.s. }
$$

Limit behaviour of cost functionals The difference of tracked and tracking cost functional has the representation

$$
\begin{aligned}
Y_{t}^{\star}-Y_{t}^{-\delta}=E & {\left[\int_{t}^{\sigma^{\delta} \wedge \tau} g\left(s, X_{s}^{\star}, Y_{s}^{\star}, Z_{s}^{\star}\right)-g\left(s, X_{s}^{-\delta}, Y_{s}^{-\delta}, Z_{s}^{-\delta}\right) d s\right.} \\
& +\left(h\left(X_{T}^{\star}\right)-h\left(X_{T}^{-\delta}\right)\right) \chi_{\sigma^{\delta} \wedge \tau=T}+a_{\tau}^{L}\left(X_{\tau}^{\star}-X_{\tau}^{-\delta}\right) \chi_{\tau<\sigma^{\delta}} \\
& \left.+a_{\sigma^{\delta}}^{U}\left(X_{\sigma^{\delta}}^{\star}-X_{\sigma^{\delta}}^{-\delta}\right) \chi_{\substack{\sigma^{\delta} \leq \tau \\
\sigma^{\delta}<T}}+\int_{\left[t, \sigma^{\delta} \wedge \tau\right)} a_{s}^{U} d C_{s}^{U} \mid \mathcal{F}_{t}\right]
\end{aligned}
$$

Again the value is monoton in $\delta$, and $\left(Y^{-\delta}, Z^{-\delta}\right)$ converges to $\left(Y^{\star}, Z^{\star}\right)$ :

Proposition 3.16. Assume that (3.10) holds. Let, for $\delta, \delta^{\prime} \in \mathbb{R}_{>0}$, the processes $Y^{-\delta}, Y^{-\delta^{\prime}}$ as defined in proposition 3.14. Then

$$
Y_{t}^{-\delta^{\prime}} \leq Y_{t}^{-\delta} \leq Y_{t}^{\star} \quad \text { for } \delta \leq \delta^{\prime} \text { for all } t \boldsymbol{P} \text {-a.s. }
$$


Proposition 3.17. Assume that (3.10) holds. Then $Y_{t}^{-\delta}$ converges to $Y_{t}^{\star} \boldsymbol{P}$ a.s. uniformly in $t$, and further

$$
\begin{aligned}
& \lim _{\delta \searrow 0} E\left[\sup _{t \leq s \leq T}\left|Y_{s}^{\star}-Y_{s}^{-\delta}\right|^{2}\right]=0, \\
& \lim _{\delta \searrow 0} E\left[\int_{t}^{T}\left|Z_{s}^{\star}-Z_{s}^{-\delta}\right|^{2} d s\right]=0 .
\end{aligned}
$$

The convergence in (3.29) is monotone.

Estimates for the difference quotient $\frac{1}{\delta}\left(Y^{\star}-Y^{-\delta}\right)$ The structure of our approach is the same as in section 3.1, but we have to make slight modifications in the definitions of upper and lower bounds $\hat{R}^{u p,-\delta}$ and $\hat{R}^{l o,-\delta}$ for the transformed process $\hat{Y}^{-\delta}$. Again, the bounds converge to a common limit $\hat{R}$ that gives an estimate for the payoff of the associated Dynkin game.

We rewrite $\frac{1}{\delta}\left(Y^{\star}-Y^{-\delta}\right)$ as uncontrolled process similar to the construction in lemma 3.11 and consider

$$
\hat{Y}_{t}^{-\delta}:=\frac{1}{\delta}\left(\left(Y_{t}^{\star}-Y_{t}^{-\delta}\right)+\int_{\left[0, \sigma^{\delta} \wedge \tau \wedge t\right)} a_{s}^{U} d C_{s}^{U}\right) \quad \text { and } \quad \hat{Z}_{t}^{-\delta}:=\frac{1}{\delta}\left(Z_{t}^{\star}-Z_{t}^{-\delta}\right) .
$$

It can be verified directly that $\left(\hat{Y}^{-\delta}, \hat{Z}^{-\delta}\right)$ is the solution of a BSDE with data

$$
\begin{aligned}
\xi^{\hat{Y}}:= & \frac{1}{\delta}\left(h\left(X_{T}^{\star}\right)-h\left(X_{T}^{-\delta}\right)\right) \chi_{\sigma^{\delta} \wedge \tau=T}+a_{\tau}^{L} \frac{1}{\delta}\left(X_{\tau}^{\star}-X_{\tau}^{-\delta}\right) \chi_{\substack{\tau<\sigma^{\delta} \\
\tau<T}} \\
& +a_{\sigma^{\delta}}^{U} \frac{1}{\delta}\left(X_{\sigma^{\delta}}^{\star}-X_{\sigma^{\delta}}^{-\delta}\right) \chi_{\sigma^{\delta} \leq \tau}+\frac{1}{\delta} \int_{\left[0, \sigma^{\delta} \wedge \tau\right)} a_{s}^{U} d C_{s}^{U} \\
g^{\hat{Y}}(t, y, z):= & \left(\Delta_{x} \hat{g}_{t} \frac{1}{\delta}\left(X_{t}^{\star}-X_{t}^{-\delta}\right)+\Delta_{y} \hat{g}_{t} \chi_{y \geq 0} \cdot y\right. \\
& \left.-\Delta_{y} \hat{g}_{t} \frac{1}{\delta} \int_{[0, t)} a_{s}^{U} d C_{s}^{U}\right) \chi_{t \leq \sigma^{\delta} \wedge \tau}+\Delta_{z} \hat{g}_{t} \cdot z \chi_{t \leq \tau} \\
C_{t}^{\hat{Y}}:= & (0,0) .
\end{aligned}
$$

Here $\Delta_{x} \hat{g}_{t}, \Delta_{y} \hat{g}_{t}$ and $\Delta_{z} \hat{g}_{t}$ are defined as:

$$
\begin{aligned}
& \Delta_{x} \hat{g}_{t}:=\left(g\left(t, X_{t}^{\star}, Y_{t}^{\star}, Z_{t}^{\star}\right)-g\left(t, X_{t}^{-\delta}, Y_{t}^{\star}, Z_{t}^{\star}\right)\right) \frac{1}{X_{t}^{\star}-X_{t}^{-\delta}} \chi_{X_{t}^{-\delta} \neq X_{t}^{\star}}, \\
& \Delta_{y} \hat{g}_{t}:=\left(g\left(t, X_{t}^{-\delta}, Y_{t}^{\star}, Z_{t}^{\star}\right)-g\left(t, X_{t}^{-\delta}, Y_{t}^{-\delta}, Z_{t}^{\star}\right)\right) \frac{1}{Y_{t}^{\star}-Y_{t}^{-\delta}} \chi_{Y_{t}^{-\delta} \neq Y_{t}^{\star}}, \\
& \Delta_{z} \hat{g}_{t}:=\left(g\left(t, X_{t}^{-\delta}, Y_{t}^{-\delta}, Z_{t}^{\star}\right)-g\left(t, X_{t}^{-\delta}, Y_{t}^{-\delta}, Z_{t}^{-\delta}\right)\right) \frac{1}{Z_{t}^{\star}-Z_{t}^{-\delta}} \chi_{Z_{t}^{-\delta} \neq Z_{t}^{\star}}
\end{aligned}
$$

Observe that $\hat{Y}^{-\delta} \geq 0$ by (3.1f) and proposition 3.16, and that, for $t \leq \sigma^{0} \wedge \tau$, $\hat{Y}_{t}^{-\delta}=\frac{1}{\delta}\left(Y_{t}^{\star}-Y_{t}^{-\bar{\delta}}\right)$.

We will assume that (3.1) holds. Again we define processes $\left(\hat{R}^{u p,-\delta}, \hat{Q}^{u p,-\delta}\right)$ and $\left(\hat{R}^{l o,-\delta}, \hat{Q}^{l o,-\delta}\right)$ as solutions to the BSDE with data $\left(\xi^{u p,-\delta}, g^{u p,-\delta}, C^{u p,-\delta}\right)$ 
and $\left(\xi^{l o,-\delta}, g^{l o,-\delta}, C^{l o,-\delta}\right)$, where

$$
\begin{aligned}
& \xi^{u p,-\delta}:=h_{x}\left(X_{T}^{x, 0, C^{L}}\right) \Gamma_{T}^{x, 0, C^{L}} \chi_{\sigma^{\delta} \wedge \tau=T}+a_{\tau}^{L} \Gamma_{\tau}^{x, 0, C^{L}} \chi_{\substack{\tau<\sigma^{\delta} \\
\tau<T}} \\
& +\left(\max _{\sigma^{0} \leq s \leq \sigma^{\delta}} a_{s}^{U}\right)\left(\sup _{\sigma^{0} \leq s \leq \sigma^{\delta}} \Gamma_{s}^{x, 0, C^{L}}\right)^{2}\left(\inf _{\sigma^{0} \leq s \leq \sigma^{\delta}} \Gamma_{s}^{x-\delta, 0, C^{L}}\right)^{-1} \chi_{\sigma^{0} \leq \tau} \\
& \xi^{l o,-\delta}:=h_{x}\left(X_{T}^{x-\delta, 0, C^{L}}\right) \Gamma_{T}^{x-\delta, 0, C^{L}} \chi_{\sigma^{0} \wedge \tau=T}+a_{\tau}^{L} \Gamma_{\tau}^{x-\delta, 0, C^{L}} \chi_{\substack{\tau<\sigma^{0} \\
\tau<T}}
\end{aligned}
$$

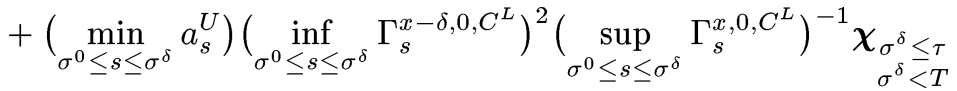

$$
\begin{aligned}
& g^{u p,-\delta}(t, y, z):=\left(g_{x}\left(t, X_{t}^{\star}, Y_{t}^{\star}\right) \Gamma_{t}^{x, 0, C^{L}}+g_{y}\left(t, X_{t}^{\star}, Y_{t}^{\star}\right) \chi_{y \geq 0} \chi_{A_{g,-\delta}^{+}} \cdot y\right. \\
& \left.+L\left(\max _{\sigma^{0} \leq s \leq \sigma^{\delta} \wedge t} a_{s}^{U}\right) \overline{C_{t}^{U,-\delta}}\right) \chi_{t \leq \sigma^{\delta} \wedge \tau}+g_{z}(t) \cdot z \chi_{t \leq \tau} \\
& g^{l o,-\delta}(t, y, z):=g_{x}\left(t, X_{t}^{-\delta}, Y_{t}^{\star}\right) \Gamma_{t}^{x-\delta, 0, C^{L}} \chi_{t \leq \sigma^{0} \wedge \tau} \\
& +\left(g_{y}\left(t, X_{t}^{-\delta}, Y_{t}^{-\delta}\right) \chi_{y \geq 0} \chi_{A_{g,-\delta}^{-}} \cdot y-L\left(\max _{\sigma^{0} \leq s \leq \sigma^{\delta} \wedge t} a_{s}^{U}\right) \overline{C_{t}^{U,-\delta}}\right) \chi_{t \leq \sigma^{\delta} \wedge \tau} \\
& +g_{z}(t) \cdot z \chi_{t \leq \tau} \\
& C_{t}^{u p,-\delta}:=C_{t}^{l o,-\delta}:=(0,0) .
\end{aligned}
$$

Here $A_{g,-\delta}^{+}$and $A_{g,-\delta}^{-}$are defined as

$$
\begin{aligned}
& A_{g,-\delta}^{+}:=\left\{t \leq \sigma^{0} \wedge \tau\right\} \cup\left\{g_{y}\left(t, X_{t}^{\star}, Y_{t}^{\star}\right) \geq 0\right\} \\
& A_{g,-\delta}^{-}:=\left\{t \leq \sigma^{0} \wedge \tau\right\} \cup\left\{g_{y}\left(t, X_{t}^{-\delta}, Y_{t}^{-\delta}\right) \leq 0\right\}
\end{aligned}
$$

We use the convention $\sup _{\sigma^{0} \leq s \leq \sigma^{\delta} \wedge t} a_{s}^{U}=0$ for $t<\sigma^{0}$. L denotes a Lipschitz constant for $g$.

The limiting process $\hat{R}$ is defined as the state process of a BSDE with data

$$
\begin{aligned}
\hat{\xi}:= & h_{x}\left(X_{T}^{x, 0, C^{L}}\right) \Gamma_{T}^{x, 0, C^{L}} \chi_{\sigma^{0} \wedge \tau=T}+a_{\tau}^{L} \Gamma_{\tau}^{x, 0, C^{L}} \chi_{\substack{\tau<\sigma^{0} \\
\tau<T}}+a_{\sigma^{0}}^{U} \Gamma_{\sigma^{0}}^{x, 0, C^{L}} \chi_{\substack{\sigma^{0} \leq \tau \\
\sigma^{0}<T}} \\
\hat{g}(t, y, z):= & \left(g_{x}\left(t, X_{t}^{\star}, Y_{t}^{\star}\right) \Gamma_{t}^{x, 0, C^{L}}+g_{y}\left(t, X_{t}^{\star}, Y_{t}^{\star}\right) \chi_{y \geq 0} \cdot y\right) \chi_{t \leq \sigma^{0} \wedge \tau} \\
& +g_{z}(t) \cdot z \chi_{t \leq \tau}
\end{aligned}
$$

and control zero. Its property as limit of the difference quotient is the subject of the following lemma, which is the analogue to lemma 3.11. The proof is straightforward.

Lemma 3.18. Assume that (2.31) and (3.1) hold. The processes $\hat{R}^{u p,-\delta}, \hat{Y}^{-\delta}$, $\hat{R}^{l o,-\delta}$ satisfy

$$
\hat{R}_{t}^{l o,-\delta} \leq \hat{Y}_{t}^{-\delta} \leq \hat{R}_{t}^{u p,-\delta} \quad \forall t \in \bar{\imath} \boldsymbol{P} \text {-a.s. }
$$

Further, $\hat{R}_{t}^{u p,-\delta}$ decreases and $\hat{R}_{t}^{\text {lo,- }-\delta}$ increases as $\delta \searrow 0$ to the same limiting process $\hat{R}$ defined above, in $L_{\mathcal{F}}^{2}(0, T ; \mathbb{R})$ and for all $t \in \bar{\imath} \boldsymbol{P}$-a.s.

We now can formulate the estimate in terms of the Dynkin game 2.5: 
Lemma 3.19. Assume that (2.31) (3.1) hold. Then

$$
\lim _{\delta \searrow 0} \frac{1}{\delta}\left(Y_{t}^{\star}-Y_{t}^{-\delta}\right) \geq R_{t}^{x}\left(\sigma^{0}, \tau\right)
$$

holds $\boldsymbol{P}$-a.s. for all $t \leq \sigma^{0} \wedge \tau$.

Proof. By lemma 3.18 it suffices to prove

$$
\hat{R}_{t} \geq R_{t}^{x}\left(\sigma^{0}, \tau\right) \quad \boldsymbol{P} \text {-a.s. for } t \leq \sigma^{0} \wedge \tau .
$$

This follows from the comparison theorem 2.14, if the data satisfy $\hat{\xi} \geq \xi^{R}$ and $\hat{g}(t, y, z) \geq g^{R}(t, y, z)$. But this is a consequence of the convexity assumptions. Especially observe that $\Gamma^{x, 0, C^{L}} \geq \Gamma^{x, 0,0}$ holds for the deflator processes, as $b$ is convex and $X^{x, 0, C^{L}} \geq X^{x, 0,0}$.

We now conclude this discussion with the

Proposition 3.20. Assume that (2.31) and (3.1) hold that there exists an optimal control $\left(C^{U}, C^{L}\right)$ for the control problem 2.2 in $\left(t_{0}, x\right)$. Let $V_{t}\left(t_{0}, x\right)$ its value. Then the lower left Dini derivative of $V$ with respect to the initial condition satisfies

$$
\Delta_{-} V_{t}\left(t_{0}, x\right) \geq u_{t}^{+}\left(t_{0}, x\right) \quad \boldsymbol{P} \text {-a.s. }
$$

Proof. By the optimality of $\left(C^{U},-C^{L}\right)$ in $\left(t_{0}, x\right)$ and lemma 3.12 we have

$$
\liminf _{\delta \searrow 0} \frac{1}{\delta}\left(V_{t}\left(t_{0}, x\right)-V_{t}\left(t_{0}, x-\delta\right)\right) \geq \liminf _{\delta \searrow 0} \frac{1}{\delta}\left(Y_{t}^{\star}-Y_{t}^{-\delta}\right) \geq R_{t}^{t_{0}, x}\left(\sigma^{0}, \tau\right)
$$

As $\tau \in \mathcal{T}_{t_{0}}$ is arbitrary this gives

$$
\Delta_{-} V_{t}\left(t_{0}, x\right) \geq \operatorname{ess} \sup _{\tau \in \mathcal{T}_{t_{0}}} R_{t}^{t_{0}, x}\left(\sigma^{0}, \tau\right) \geq u_{t}^{+}\left(t_{0}, x\right) \quad \boldsymbol{P} \text {-a.s }
$$

\subsection{Proof and discussion}

Proof of theorem 3.1. By proposition 3.20, the definitions in (2.6), proposition 3.13 and theorem 2.23 we have the relations

$$
\Delta^{+} V_{t}\left(t_{0}, x\right) \leq u_{t}^{-}\left(t_{0}, x\right) \leq u_{t}^{+}\left(t_{0}, x\right) \leq \Delta_{-} V_{t}\left(t_{0}, x\right) \leq \Delta^{+} V_{t}\left(t_{0}, x\right) .
$$

and indeed, equality holds in 3.35. So $V$ is differentiable at $x$ with partial derivative equal to the solution of Isaac's equation $u_{t}^{-}\left(t_{0}, x\right)=u_{t}^{+}\left(t_{0}, x\right)=$ $u_{t}\left(t_{0}, x\right)$.

Let us suppress dependence on $\left(t_{0}, x\right)$. By (3.22), (3.34), (3.35) and the Isaac's equation

$$
\begin{aligned}
& \operatorname{essinf}_{\sigma \in \mathcal{T}} R_{t}\left(\sigma, \tau^{0}\right)=\operatorname{ess~sup}_{\tau \in \mathcal{T}} \operatorname{essinf}_{\sigma \in \mathcal{T}} R_{t}(\sigma, \tau) \\
& =u_{t}=\operatorname{essinf}_{\sigma \in \mathcal{T}} \operatorname{ess}_{\sup _{\tau \in \mathcal{T}}} R_{t}(\sigma, \tau)=\operatorname{ess}_{\sup } \operatorname{su}_{\tau \in \mathcal{T}} R_{t}\left(\sigma^{0}, \tau\right) .
\end{aligned}
$$

Hence $\left(\sigma^{0}, \tau^{0}\right)$ is an optimal pair or saddle point for the associated Dynkin game. 
Statements of the type of theorem 3.1 make it possible to transfer results on Dynkin games to singular control problems and vice versa. In general the former are better accessible by analytical arguments than the latter, whereas the space of controls is more easily treated with topological arguments. The transferable results include questions of existence - or nonexistence - of optimal policies, structure of solutions and properties of the value functions.

In the general formulation of definition 2.2, the associated Dynkin game depends in its data on the value of the control problem. This is due to the nonlinearity of the BSDE in (2.2) in $Y$. In economic terms, this nonlinearity corresponds to a nonadditive stochastic differential utility as introduced by DUfFIE and EPSTEIN [19]. Its main feature is that, in contrast to standard additive utility induced by linear BSDE, preferences with respect to timing differences are not induced by discounting.

For problems with a Markovian structure and suitably smooth data solutions of the Dynkin game have the structure of continuation and stopping regions for the trajectory of the state process. A player intervenes when the state process hits a specific stopping region. In the complement of the stopping regions the game continues up to a terminal time $T$. In singular control problems solutions often exhibit a similar structure, where no control is exercised as long as the optimal trajectory remains within a no-transaction-region. An optimal control is "bang-bang", i.e. it intervenes only to prevent the state from exiting this region, thus creating a reflected state process. In this context theorem 3.1 hints that the no-control region in the control problem and the continuation region in the Dynkin game should agree.

An analytical study of the value function of the control problem involves the treatment of variational inequalities derived from degenerate Hamilton-JacobiBellman PDE's. The aim is to formulate a PDE with suitable boundary conditions, such that a solution can be identified as the value function through a verification theorem, with the boundary separating control and no-control regions. An important condition at the unknown boundary is the smooth pasting assumption which requires that the value function be twice differentiable in the space variable. This assumption can be justified by theorem 3.1 and the principle of smooth fit that occurs in problems of optimal stopping through their well established relation with Stefan or free boundary value problems.

Equivalence of optimal stopping and control It is also possible to take the route backwards in our pathwise approach and construct optimal controls from a set of optimal stopping times for different starting points. This equivalence of optimal stopping and singular stochastic control was established by Baldursson and Karatzas [6] and Boetius and Kohlmann [12] for monotone control. The basic idea is to construct impulse controls for a finite set of initial states and optimal stopping times. These impulse controls then form a convergent sequence with an optimal control process as limit. This constructive approach makes it possible to further investigate the structure of optimal controls and exhibits their characterization as local times. We plan to establish this equivalence in the general situation of (2.2) in our future work.

Problems with finite fuel It was shown in the case of Brownian motion and monotone control by CHOw, Menaldi and RoBin [13] with analytical and 
Karatzas [34] with probabilistic arguments, and by Karatzas and Shreve [37] for bounded variation control, that the statements of theorem 3.1 remain essentially unchanged if an additional finite fuel condition is imposed on the admissible controls. Under a natural generalization of the finite fuel condition this property is preserved in more general situations. This was carried out by Boetius and Kohlmann [11], [12]. One characteristic is the fact that optimal controls behave like optimal controls in the unrestricted problem, until they run out of fuel and the state process rests on the "boundary" representing the restriction. In the general formulation, this boundary is itself solution of the uncontrolled forward equation. The only minor problem in extending these results to our situation is to show that the tracking processes are still admissible.

On the contrary, under a strict finite fuel condition $|C|_{T}<K$ there may be a timing value of control and optimal strategies are not necessarily truncated copies of strategies in the unrestricted case. This is clear from the heuristic argument, that the relative displacement caused by a given impulse varies with time. Intuitively, if $b_{x}>0$ then optimal controls will use up resources faster than in the unrestricted case, as the possible relative impact of a unit of resources deminishes as time increases. Saving more resources than the unrestricted strategy will be optimal only if $b_{x}<0$.

Both concepts, strict and generalized finite fuel, coincede in the case of a monotone or bounded variation follower for Brownian motion, i.e. if $X_{t}^{x, 0,0}=$ $x+W_{t}$.

Infinite time horizon The treatment of problems where the terminal time is a possibly infinite stopping time is not different in principle. It is important to ensure validity of comparison theorems and a priori estimates, for which one may consult e.g. El KARoui and Quenez [24]. Additional requirements are integrability conditions for the data.

Related topics in BSDE theory Backward equations with a formal similarity to (2.2) appear in the context of $g$-semisolutions and $g$-semimartingales, and reflected backward stochastic differential equation (RBSDE).

The notion of $g$-supersolutions and $g$-supermartingales was introduced by PENG [49], [48] in his study of an extended concept of expectation to match nonadditive utility frameworks.

The study of BSDE with a reflecting boundary was initiated, to our knowledge, by El Karoui, Kapoudjian, Pardoux, Peng, Quenez [21] and extended to the case of reflection at two boundaries by CVITANIć and KARATZAS [14]. Further investigations into the subject and its applications in finance where carried out by El Karoui and Quenez [24], Hamadene, Lepeltier and Matoussi [29], Hamadene, Lepeltier and Peng [30], Hamadene and LEPELTIER [28] and MA and YoNG [42] for higher dimensions. A strong motivation for the study of reflected BSDE is that they allow to model sellers prices for american contingent claims. One of the most interesting properties is that the state process of a solution to a RBSDE is also the value of an optimal stopping problem, and the optimal stopping time can be determined as first action time of the control causing the reflection.

Given the structure of solutions to the control problem in smooth situations and typical characterizations of the value function through variational inequal- 
ities it is fairly straight forward to suggest that the value process $V$ itself be solution to a suitable RBSDE; however determining the reflecting boundaries is part of the solution to the control problem.

We exploit possible links between control problems and RBSDE in the next section.

\section{Historical remarks}

In its basic struture, the probabilistic approach to singular control taken here is due to Karatzas and Shreve ([35], [36]) and was extended in [6] and [12]. However, the problems studied therein have in essence monotone controls, and associated a one player optimal stopping problem in contrast to our situation. Investigation of a connection with a Dynkin game was motivated by the relation between double barrier RBSDE and Dynkin games established by CVITANIĆ and KARATZAS [14].

The idea for the removal of control applied in the proofs of lemmata 3.11, 3.18 is taken from PENG [49]. 


\section{Characteristic RBSDE of a singular control problem}

Consider the following stochastic system in integral form:

$$
\begin{aligned}
A_{t}= & h_{x}\left(X_{T}^{t_{0}, x, 0,0}\right) \Gamma_{T}^{t_{0}, x}+\left(K_{T}^{U}-K_{t}^{U}\right)-\left(K_{T}^{L}-K_{t}^{L}\right) \\
& +\int_{t}^{T}\left\langle D g\left(s, X_{s}^{t_{0}, x, 0,0}, V_{s}\left(t_{0}, x\right)\right),\left(\Gamma_{s}^{t_{0}, x}, A_{s}, B_{s}\right)\right\rangle d t \\
& -\int_{t}^{T} B_{s}^{\top} d W_{s} \\
a_{t}^{L} \Gamma_{t}^{t_{0}, x} \leq & A_{t} \leq a_{t}^{U} \Gamma_{t}^{t_{0}, x} \\
0= & \int_{t_{0}}^{T}\left(a_{s}^{U} \Gamma_{s}^{t_{0}, x}-A_{s}\right)^{+} d K_{s}^{U}=\int_{t_{0}}^{T}\left(A_{s}-a_{s}^{L} \Gamma_{s}^{t_{0}, x}\right)^{+} d K_{s}^{L}
\end{aligned}
$$

Definition 4.1. A triple $(A, B, K) \in\left(L_{\mathcal{F}}^{2}(0, T ; \mathbb{R}) \times L_{\mathcal{F}}^{2}\left(0, T ; \mathbb{R}^{d}\right) \times \mathcal{A}\right)$ satisfying (4.1) for $t_{0} \leq t \leq T \boldsymbol{P}$-a.s. is called a solution to the RBSDE (4.1) on $\left[t_{0}, T\right]$.

Dependence on $t_{0}, x$ is noted by a superscript, but we continue to suppress dependence on $t_{0}$. Observe that the process $K=\left(K^{U},-K^{L}\right)$ prevents $A_{t}$ from leaving the interval $\left[a_{t}^{L} \Gamma_{t}^{x}, a_{t}^{U} \Gamma_{t}^{x}\right]$ acting only on its boundary; it is this property that the notion of "reflection" stems from.

Recall once more the stochastic game as in definition 2.5. The following is an adaption of Cvitanić and Karatzas [14], Theorem 4.1 or Ma and Yong [42], Theorem 8.6.1 to our situation:

Theorem 4.2. Suppose there exists a solution $(A, B, K)$ to the RBSDE (4.1). Then the Dynkin game (2.7) has a solution. Its value, which solves the Isaac's equation 2.7, is given as $u_{t}(x)=A_{t}^{x}$, and the pair of stopping times

$$
\begin{aligned}
\hat{\sigma}_{t} & :=\inf \left\{s \in[t, T) \mid A_{s}^{x}=a_{s}^{U} \Gamma_{s}^{x}\right\} \\
\hat{\tau}_{t} & :=\inf \left\{s \in[t, T) \mid A_{s}^{x}=a_{s}^{L} \Gamma_{s}^{x}\right\}
\end{aligned}
$$

is a saddle point for the Dynkin game.

A proof can be found in [14].

Corollary 4.3. Assume that (2.31) and (3.1) hold and that there exists an optimal control $C=\left(C^{U},-C^{L}\right)$ for the control problem 2.2 at the starting point $\left(t_{0}, x\right)$. Assume further that (4.1) has a solution $\left(A^{t_{0}, x}, B, K\right)$ on $\left[t_{0}, T\right]$. Then

$$
A_{t}^{t_{0}, x}=\frac{\partial}{\partial x} V_{t}\left(t_{0}, x\right) \quad \forall t \in\left[t_{0}, T\right] \boldsymbol{P} \text {-a.s. }
$$

This is an immediate consequence of theorems 3.1 and 4.2.

The obvious similarities in the derivation of a pair of optimal stopping times from solutions to the control problem (2.3) and the RBSDE (4.1) exhibited in theorems 3.1 and 4.2 lead us to the following

\section{Hypothesis 4.4. (Equivalence of control problem and RBSDE)}

1. Assume that for fixed $t_{0}$ and for all $x$ the RBSDE (4.1) has a solution $(A, B, K)$. Then there exists an optimal control in the control problem, and the value functions and state process satisfy (4.3). 
2. Assume that (2.31) and (3.1) hold and that there exists an optimal control $C=\left(C^{U},-C^{L}\right)$ for the control problem 2.2 at the starting point $\left(t_{0}, x\right)$. Then the RBSDE (4.1) has a solution $\left(A^{t_{0}, x}, B, K\right)$, and (4.3) holds.

Discussion of hypothesis 4.4. If (4.1) has a solution $(A, B, K)$ for all $x$, then the family of Dynkin games for initial conditions $x$ has a (family of) solutions. As indicated in the discussion of equivalence of optimal stopping and singular stochastic control, with a constructive approach we can find the relation (3.2) and an optimal control, hence (4.3) holds by corollary 4.3.

Actually the equivalence of optimal stopping and singular stochastic control remains an open question at present, but we suggest that an extension of the results in [12] should be possible.

Now consider the second statement. We suggest that it should be possible to construct a solution to the RBSDE from a family of solutions to the associated Dynkin games. The conclusion of the hypothesis then follows from corollary 4.3 .

Note that the optimal control $C$ and the reflection process $K$ will not agree. This is an immediate consequence of the fact that in many practical situations with smooth data $C$ is singular, i.e. not absolutely continuous with respect to Lebesgue measure. This is a consequence of the characterization of optimal controls through Brownian local time. On the contrary, the process $K$ causing the reflection is absolutely continuous with respect to Lebesgue measure, as the information process $B$ already absorbs the strong irregularity caused by the release of Brownian information. So both processes $C$ and $K$ are somewhat different in nature. However, formulae (3.8), (3.27) and (4.2) suggest that their points of increase agree.

For properties of $C$ see Boetius and Kohlmann [12], for those of $K$ consult e.g. El Karoui and Quenez [24]. 


\section{References}

[1] M. Alario-Nazaret, J. Lepeltier, and B. Marchal, Dynkin games, in Lecture Notes in Control and Inform. Sci., vol. 43, Springer, Berlin, 1982, pp. $23-42$.

[2] L. H. R. Alvarez, A class of solvable singular stochastic control problems, Stochast. Stochast. Rep., 67 (1999), pp. 83 - 122.

[3] F. Antonelli, Backward-forward stochastic differential equations, Ann. Appl. Prob., 3 (1993), pp. 777 - 793.

[4] H. Arntzen, Otpimal choice of production capacity in a random market, Stoch. Stoch. Proc., 55 (1995), pp. $87-120$.

[5] F. Baldursson, Singular stochastic control and optimal stopping, Stochastics, 21 (1987), pp. 1 - 40.

[6] F. Baldursson AND I. Karatzas, Irreversible investment and industry equilibrium, Finance Stochast., 1 (1997), pp. 69 -89.

[7] J. Bather And H. Chernoff, Sequential decisions in the control of a spaceship, in Proc. Fifth Berkeley Symposium on Mathematical Statistics and Probability, vol. 3, 1966, pp. $181-207$.

[8] V. Beneš, L. Shepp, And H. Witsenhausen, Some solvable stochastic control problems, Stochastics, 4 (1980), pp. $39-83$.

[9] A. Bensoussan and A. Friedman, Non-linear variational inequalities and differential games with stopping times, J. Funct. Anal., 16 (1974), pp. $305-352$.

[10] J. Bismut, Sur un problème de dynkin, Z.f.W., 39 (1977), pp. 31 - 53.

[11] F. Boetius, Zusammenhang zwischen optimalem Stoppen und singulärer Kontrolle, Diplomarbeit, Universität Konstanz, 1996.

[12] F. Boetius and M. Kohlmann, Connections between optimal stopping and singular stochastic control, Stoch. Proc. Appl., 77 (1998), pp. $253-$ 281.

[13] P.-L. Chow, J.-L. Menaldi, And M. Robin, Additive control of stochastic linear systems with finite horizon, SIAM J. Control Optim., 23 (1985), pp. $858-899$.

[14] J. Cvitanić And I. Karatzas, Backward stochastic differential equations with reflection and Dynkin games, Annals of Prob., 24 (1996), pp. 2024 2056.

[15] - Hedging and portfolio optimization unter transaction costs: A martingale approach, Math. Finance, 6 (1996), pp. $133-165$.

[16] J. Cvitanić, H. Pham, AND N. Touzi, A closed-form solution to the problem of super-replication under transaction costs, Finance Stochast., 3 (1999), pp. $35-54$. 
[17] M. Davis And A. Norman, Portfolio selction with transaction costs, Math. Oper. Res., 15 (1990), pp. 676 - 713.

[18] A. Dixit And R. Pindyck, Investment under Uncertainty, Princeton Univ. Press, Princeton, 1994.

[19] D. Duffie And L. G. Epstein, Stochastic differential utility (appendix c with Costis Skiadas, Econometrica, 60 (1992), pp. 353 - 394.

[20] E. Dynkin And A. Yushkevich, Theorems and Problems in Markov Processes, Plenum Press, New York, 1968.

[21] N. El Karoui, C. Kapoudjian, E. Pardoux, S. Peng, and M.-C. QUENEZ, Reflected solutions of backward SDE's, and related obstacle problems for PDE's, Annals of Prob., 25 (1997), pp. $702-737$.

[22] N. El Karoui and I. Karatzas, Probabilistic aspects of finite-fuel, reflected follower problems, Acta Appl. Math., 11 (1988), pp. 223 - 258.

[23] — A new approach to the skorohod problem, and its applications, Stochast. Stochast. Rep., 34 (1991), pp. $57-82$.

[24] N. El Karoui AND M. Quenez, Non-linear pricing theory and backward stochastic differential equations, in Financial Mathematics, Lectures given at the 3rd Session of the Centro Internazionale Matematico Estivo (C.I.M.E.) held in Bressanone, Italy, July 8-13, 1996, W. Runggaldier, ed., Springer, 1997, pp. $191-246$.

[25] W. Fleming and H. Soner, Controlled Markov Processes and Viscosity Solutions, Springer, New York, Berlin, Heidelberg, 1993.

[26] A. Friedman, Stochastic Differential Equations and Applications, vol. 2, Academic Press, New York, San Francisco, London, 1976.

[27] S. Hamadène and J.-P. LePeltier, Zero-sum stochastic differential games and backward equations, Systems Control Letters, 24 (1995), pp. 259 -263 .

[28] - Reflected BSDEs and mixed game problem, Stoch. Proc. Appl., 85 (2000), pp. $177-188$.

[29] S. Hamadene, J.-P. Lepeltier, and A. Matoussi, Double barrier backward SDEs with continuous coefficients, in Backward stochastic differential equations, N. El Karoui and L. Mazliak, eds., Addison Wesley Longman, 1997, pp. $161-176$.

[30] S. Hamadene, J.-P. Lepeltier, and S. Peng, BSDEs with continuous coefficients and stochastic differential games, in Backward stochastic differential equations, N. El Karoui and L. Mazliak, eds., Addison Wesley Longman, 1997, pp. $115-128$.

[31] N. Ikeda And S. Watanabe, Stochastic Differential Equations and Diffusion Processes, North-Holland, Amsterdam, Oxford, New York, 2 ed., 1989 . 
[32] I. KaRATZAS, The monotone follower problem in stochastic decision theory, Appl. Math. Optim., 7 (1981), pp. 175 - 189.

[33] _ - A class of singular stochastic control problems, Adv. Appl. Prob., 15 (1983), pp. $225-254$.

[34] - Probabilistic aspects of finite fuel stochastic control, Proc. Natl. Acad. Sci., 82 (1985), pp. 5579 - 5581.

[35] I. Karatzas and S. Shreve, Connections between optimal stopping and singular stochastic control I. Monotone follower problems, SIAM J. Control Optim., 22 (1984), pp. 856 - 877.

[36] - Connections between optimale stopping and singular stochastic control II. Reflected follower problems, SIAM J. Control Optim., 23 (1985), pp. $433-451$.

[37] _ Equivalent models for finite-fuel stochastic control, Stochastics, 18 (1986), pp. $245-276$.

[38] — Brownian Motion and Stochastic Calculus, no. 113 in GTM, Springer, New York, 3 ed., 1994.

[39] T. . KoBILA, A class of solvable stochastic investment problems involving singular stochastic control, Stochast. Stochast. Rep., 43 (1993), pp. 29 63.

[40] N. Krylov, Controlled Diffusion Processes, Springer, New York, Berlin, Heidelberg, 1980.

[41] J. Ma, P. Protter, And J. Yong, Solving forward-backward stochastic differential equations explicitly - a four step scheme, Probab. Theory Related Fields, 98 (1994), pp. 339 - 359.

[42] J. Ma And J. Yong, Forward-Backward Stochastic Differential Equations and Their Applications, Springer, Berlin, Heidelberg, New York, 1999.

[43] R. McDonald and D. Siegel, The value of waiting to invest, The Quarterly J. of Economics, 101 (1986), pp. 707 - 727.

[44] J. Menaldi and M. TaksaR, Optimal correction problem of a multidimensional stochastic system, Automatica, 25 (1989), pp. 223 - 232.

[45] H. Mовімото, Dynkin games and martingale methods, Stochastics, 13 (1984), pp. $213-228$.

[46] J. Neveu, Discrete-Parameter Martingales, North Holland, Amsterdam, 1975.

[47] E. Pardoux and S. Peng, Adapted solution of a backward stochastic differential equation, Systems and Control Letters, 14 (1990), pp. 55-61.

[48] S. Peng, Backward SDE and g-expectation, in Backward stochastic differential equations, N. El Karoui and L. Mazliak, eds., Addison Wesley Longman, 1997, pp. $141-159$. 
[49] - Monotonic limit theorem of BSDE and nonlinear decomposition theorem of Doob-Meyer's type, Probab. Theory Related Fields, 113 (1999), pp. $473-499$.

[50] R. PINDYCK, Irreversibility and the explanation of investment behaviour, in Stochastic Models and Option Values, D. Lund and B. Øksendal, eds., North Holland, Amsterdam, 1991, pp. 129 - 141.

[51] P. Protter, Stochastic Integration and Differential Equations, Springer, Berlin, Heidelberg, New York, 1990.

[52] A. Shiryayev, Optimal Stopping Rules, Springer, New York, Heidelberg, Berlin, 1978.

[53] S. Shreve, An introduction to singular stochastic control, in Stochastic Differential Systems, Stochastic Control Theory and Applications, W. Fleming and P.-J. Lions, eds., Springer, 1988, pp. 513 - 528.

[54] S. Shreve, J. Lehotzky, and D. Gaver, Optimal consumption for general diffusions with absorbing and reflecting barriers, SIAM J. Control Optim., 22 (1984), pp. $55-75$.

[55] H. Soner And S. Shreve, Regularity of the value function for a twodimensional singular stochastic control problem, SIAM J. Control Optim., 27 (1989), pp. 876 - 907.

[56] H. Soner, S. Shreve, And J. Cvitanić, There is no nontrivial hedging portfolio for option pricing with transaction costs, Ann. Appl. Probab., 5 (1995), pp. $327-355$.

[57] L. STETTNER, Zero-sum markov games with stopping and impulsive strategies, Appl. Math. Optim., 9 (1982), pp. 1 - 24.

[58] J. Yong And X. Y. Zhou, Stochastic Controls: Hamiltomian Systems and HJB Equations, Springer, New York, 1999. 